\title{
Phytosociological and ecological study of springs in Trentino (south-eastern Alps, Italy)
}

\author{
Marcello TOMASELLI*, Daniel SPITALE ${ }^{1)}$ and Alessandro PETRAGLIA \\ University of Parma, Department of Evolutionary and Functional Biology, Viale G.P. Usberti 11/A, 43100 Parma (Italy) \\ ${ }^{1)}$ Museo Tridentino di Scienze Naturali, Limnology and Phycology Section, Via Calepina 1438122 Trento (Italy) \\ *e-mail corresponding author: marcello.tomaselli@unipr.it
}

\section{ABSTRACT}

A phytosociological survey of the crenic vegetation was made in Trentino (south-eastern Alps), combining the field method of the Braun-Blanquet approach with a numerical syntaxonomical analysis. A set of 139 phytosociological relevés, including vascular plants and bryophytes, were classified using cluster analysis. The vegetation types were assigned to 7 different phytosociological classes: Platyhypnidio-Fontinalietea antipyreticae, Montio-Cardaminetea, Adiantetea, Scheuchzerio-Caricetea nigrae, MolinioArrhenatheretea, Galio-Urticetea, Mulgedio-Aconitetea. The classes Platyhypnidio-Fontinalietea antipyreticae and MontioCardaminetea represent the core of crenic vegetation, including permanently or intermittently submerged plant communities, mostly made up of bryophytes, or non-submerged communities dominated by bryophytes or vascular plants. The other classes include chasmophytic bryophyte-rich communities and hygrophilous or tall herb communities lying around the periphery of the springs. 23 vegetation types were identified and, whenever possible, classified at the association level, or as phytocoena. The environmental parameters showed different ranges among vegetation types. A number of environmental variables were recorded during the vegetation survey, including altitude, shading, discharge, flow velocity, with exhaustive hydrochemical sampling. Conductivity, alkalinity and $\mathrm{pH}$ showed similar distribution patterns, clearly separating the vegetation types into two distinct groups, differing in the nature of the substratum. The altitudinal range was very broad and shading was also very variable. Nitrate and phosphate levels showed that the majority of vegetation types were irrigated by oligotrophic crenic waters. Finally, the bryophyte-dominated vegetation types belonging to the class Platyhypnidio-Fontinalietea antipyreticae occurred in springs with the highest discharge values and variation. Discriminant analysis confirmed that the environmental descriptors that best accounted for the among-group variation were $\mathrm{pH}$ and alkalinity (first variate); the second variate reflected a gradient of altitude and discharge.

Key words: crenon, vegetation, phytosociology, numerical syntaxonomy

\section{INTRODUCTION}

Interest in the description of crenic vegetation depends mainly on its very peculiar floristic composition (Zechmeister \& Mucina 1994). The bulk of the crenic flora in montane regions is, in fact, composed of species adapted to particular habitat conditions, such as constant low water temperature, high air humidity (also in summer) and high oxygen saturation, due to the frequently turbulent water flow (Cantonati et al. 2006). In particular, the constancy of thermal conditions in this habitat leads to the establishment of stenothermic plants, many being relicts of past climatic periods, that survive in this typical refugial habitat (Wilmanns 1989).

Crenic vegetation is typically composed of a mixture of vascular plants and bryophytes, with the former prevailing in shaded sites at lower altitudes, and the latter dominating the communities of open habitats, from the subalpine to alpine vegetation belts (Zechmeister \& Mucina 1994; Spitale et al. 2009).

Vegetation diversity in springs is primarily determined by a combination of interacting physical and chemical factors. Of the physical factors, solar radiation, water temperature, and current flow velocity play a major role in determining floristic diversity and struc- ture in crenic vegetation (Hinterlang 1992). The most important hydrochemical factors are $\mathrm{pH}$, water hardness, oxygen saturation and nutrient content (Warnke \& Bogenrieder 1985). Duration of snow cover, which affects duration of the growing season, can also play an important role in conditioning crenic vegetation diversity at high altitudes (Zechmeister \& Mucina 1994).

Despite its undisputable floristic and ecological interest, very few geobotanists and plant ecologists have focussed their attention on crenic vegetation. Maas (1959) produced an historical, comprehensive monograph on the crenic vegetation of Central Europe. In the Alps, crenic vegetation has mostly been sampled and characterised within comprehensive surveys of particular areas, where its contribution is limited (Braun-Blanquet \& Braun-Blanquet 1931; Guinochet 1938; BraunBlanquet 1954; Giacomini \& Pignatti 1955; Wikus 1960; Giacomini et al. 1962; Boiti et al. 1989; Herter 1990; Theurillat 1992; Gerdol 1994; Dirnböck et al. 1999). Moreover, the definition and classification of crenic vegetation types is uneven across these accounts. Very few authors have published regional studies centred on bryophyte-dominated crenic vegetation (Hébrard 1973; Miserere \& Buffa 2001). The only general monograph devoted to the springs of the Alps is that of 
Geissler (1976), however this is restricted to sites within the alpine vegetation belt.

This paper aims to fill this gap by providing a regional survey focused on the crenic vegetation of part of the south-eastern Italian Alps. For the first time, a systematised sampling technique is applied across the crenic vegetation, along an altitudinal gradient from the collinar to the alpine vegetation belt. The data on physical and chemical variables collected in the field were primarily used to characterise the vegetation types from an ecological viewpoint. We also tried to identify the major environmental determinants of crenic vegetation, which control crenic floristic diversity in the area.

\section{METHODS}

\subsection{The study area}

The research was conducted in the Autonomous Province of Trento (the geographic name of the area is Trentino), located in the south-eastern Italian Alps. More than 100 springs, representing habitat variability in the area, were identified on the basis of specific and standardized requirements, such as perennial flow and naturalness (no man-made water-collection systems). 81 springs, distributed homogenously throughout the Province, were selected for the study. All the morphological types distinguished by Thienemann (1922), from seepages and tufa springs to large karstic rheocrenes, were sampled. The springs were located over a wide altitudinal range (from 170 to $2792 \mathrm{~m}$ a.s.1.), from the collinar to the nival vegetation belt, and on a variety of lithologies, from limestones and dolomites, to siliceous rocks (metamorphic, microcrystalline, crystalline). A more detailed description of the whole environmental dataset can be found in Spitale et al. (2009).

\subsection{Field sampling}

Vegetation in the 81 selected springs was sampled systematically according to the phytosociological method (Braun-Blanquet 1964; Westhoff \& van der Maarel 1973). Sampling was conducted once at all sites during the summers of 2005 and 2006. 139 relevés were taken in relatively uniform stands, whose delimitation was the first critical step. As explained in Tomaselli (2007), we defined "spring area" as the surface influenced by the water flowing from the spring (i.e. the surface covered by flushing water and the area reached by water spray). Within this area, we identified homogeneous stands in which both floristic composition and structure were relatively uniform. Because springs frequently have a mosaic structure, we ensured that we sampled all relevant homogeneous patches inside the spring area. For each phytosociological relevé a visual estimate of the cover-abundance of each species was carried out according to the Braun-Blanquet scale: $r$ (one or few individuals with negligible cover); + (occasional individuals and $<1 \%$ cover $) ; 1$ ( $1-5 \%$ cover $) ; 2$
( $>5-25 \%)$ cover; 3 ( $>25-50 \%$ cover); class 4 ( $>50-75 \%$ cover); class $5(>75-100 \%)$.

The geographical coordinates and altitude of each spring were recorded by GPS (Garmin GPSMAP 76S). Temperature was recorded in the field using a digital probe, and the highest current velocity was measured with an OTT propeller flow meter. In large springs the velocity/area method (e.g., Gordon et al. 2004) was applied, whereas in small springs discharge estimates were made using graduated containers and a chronometer. Water for hydrochemical analyses (including the measurement of $\mathrm{pH}$, conductivity and nutrients) was collected in acid-cleaned graduated bottles, following the standard methods of the American Public Health Association (APHA 2000). As indicator of shading, canopy was estimated visually in four classes: $1=0 \div$ $25 \%, 2=25 \div 50 \%, 3=50 \div 75 \%$, and $4=75 \div 100 \%$ (Spitale 2007).

\subsection{Vegetation analysis}

The original 139 vegetation relevés were first classified into phytosociological classes, according to the species fidelity principle (Braun-Blanquet 1964). The bulk of the original set (127 relevés) was then divided into two subsets. These correspond to permanently or intermittently submerged plant communities mostly made up of bryophytes (Platyhypnidio-Fontinalietea antipyreticae class), and to non-submerged, or only occasionally submerged, plant communities dominated by bryophytes or vascular plants (Montio-Cardaminetea class). The residual 12 relevés form a further small, heterogeneous subset including chasmophytic bryophyterich communities and hygrophilous and tall herb communities around the inner margins of the springs. Both subsets were subjected to numerical classification to obtain clusters of relevés. The Braun-Blanquet coverabundance estimates for all species of every relevé were transformed according to van der Maarel (1979). The clustering procedure used the incremental sum of squares based on the chord distance. Computations were performed using SYN-TAX 2000 (Podani 2001).

The clusters obtained were considered as local vegetation types to be compared with the Braun-Blanquet syntaxa. Comparisons were based both on local monographic studies (Gerdol \& Tomaselli 1988; 1997; Marstaller 1987; Schmidt 1993; Privitera \& Puglisi 2004) and more general vegetation surveys covering broader areas (Geissler 1976; Philippi \& Oberdorfer 1992; Grabherr \& Mucina 1993; Mucina et al. 1993; Zechmeister 1993; Zechmeister \& Mucina 1994). The list of diagnostic vascular species was compiled from these authors, whereas that for bryophytes was integrated from Dierßen (2001).

Several relevé clusters were evaluated as independent vegetation units, but they could not be referred to an association, because the dominant species had weak diagnostic power and no other species could be retained 
as characteristic. We decided to classify each relevé cluster as a phytocoenon following the suggestion of Kopecký \& Hejný (1978). The different phytocoena were designated by the names of the dominant species.

Species nomenclature follows Aeschimann et al. (2004) for vascular plants, Hill et al. (2006) for mosses and Ros et al. (2007) for liverworts. Nomenclature of the syntaxa follows Zechmeister (1993) and Zechmeister \& Mucina (1994) and is in accordance with the rules of the "Code of Phytosociological Nomenclature" (Weber et al. 2000).

\subsection{Ecological inferences}

Variation ranges of environmental parameters within the vegetation types were tabulated, indicating median, lower and upper quartiles. To determine the extent to which a set of environmental variables explained the phytosociological grouping, we performed a discriminant analysis (Legendre \& Legendre 1998). We first tested the different functions for statistical significance, retaining only significant functions for further examination. Significance was tested (1) by computing the Wilks lambda (which assumes values of $0=$ perfect discrimination, to $1=$ no discrimination) and (2) by a chisquare statistic. To interpret the discriminant functions we reported the structure coefficients for each variable for each significant function. The factor structure coefficients are the correlations between the variables in the model and the discriminant functions. Finally, in order to determine which groups are discriminated by respective functions, we projected the group centroids in the canonical discriminant space. Only associations and phytocoena that occurred at least twice were used for the analysis.

\section{RESULTS}

\subsection{Vegetation analysis}

\subsubsection{Submerged bryophyte vegetation (Platyhypnidio-} Fontinalietea antipyreticae)

A first classification dendrogram of 34 phytosociological relevés is shown in figure 1a. It includes the phytosociological relevés inside stands of permanently or intermittently submerged plant communities, almost exclusively composed by bryophytes.

All the relevés in the dendrogram can be incorporated within the class Platyhypnidio-Fontinalietea antipyreticae, comprising bryophyte communities forming a submerged stratum within fast-flowing springs, from the collinar to the montane vegetation belt. Five different communities were recorded in the study area.

\section{Scapanietum undulatae (SU - Tab. 1)}

Cluster SU includes 4 relevés where Fontinalis antipyretica (generally dominant) and Scapania undulata are the only constant bryophytes. Few other bryophythes occur with any frequency and, of them, only Pla- giochila porelloides attains a measure of prominence. Vascular plants are few in number and typically of low cover.
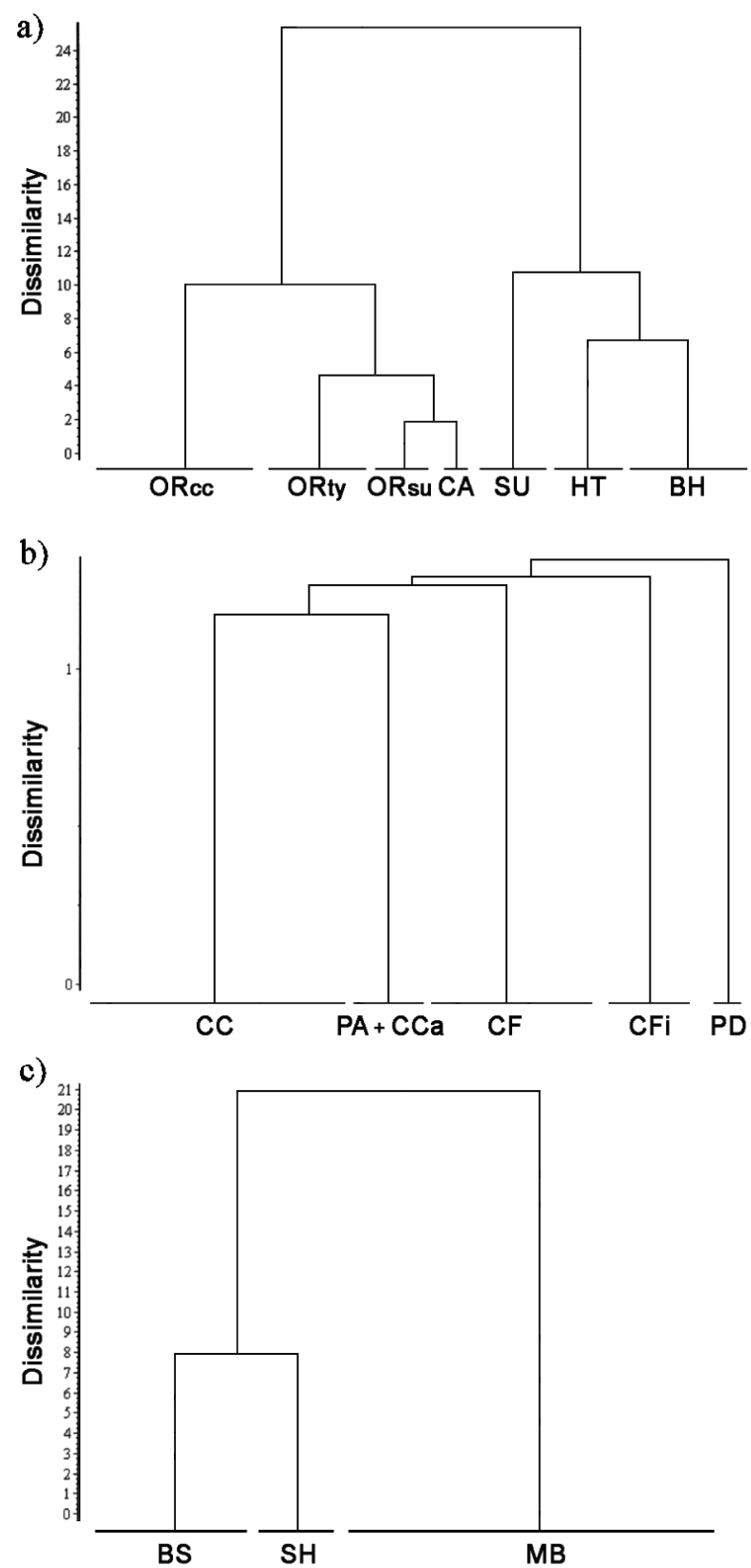

Fig. 1. Dendrogram of vegetation types: a) class Platyhypnidio-Fontinalietea antipyreticae; b) alliance Cratoneurion commutati; c) alliance Cardamino-Montion. Acronyms as in the text.

The plant community is comparatively close to the Scapanietum undulatae subass. fontinalietosum antipyreticae, owing to the constancy of both the character species of the association (Scapania undulata) and the differential species of the subassociation (Fontinalis antipyretica). Relevé 4 is differentiated by the domi- 
nance of Plagiochila porelloides and by the prominence of Porella platyphylla (variant of Plagiochila porelloides).

In the study area, the association is the only representative of the alliance Racomitrion acicularis and of the order Brachythecietalia plumosi, including the acidophilous communities dominated by submerged bryophytes on siliceous substrata, from the collinar to the subalpine vegetation belt.

The Scapanietum undulatae usually occurs within montane springs, irrigated strongly by base-poor and oligotrophic waters. The subassociation fontinalietosum antipyreticae and the variant of Plagiochila porelloides correspond to somewhat more disturbed habitats with nutrient-enriched (mostly nitrogen) crenic waters.

\section{Oxyrrhynchietum rusciformis (OR - Tab. 2)}

Cluster OR includes 18 relevés in which Platyhypnidium riparioides is the only constant bryophyte, almost always dominant or codominant. The only other, relatively frequent, bryophyte is the moss Palustriella commutata, which is sometimes so abundant as to be codominant or dominant. Other locally prominent bryophytes are Brachythecium rivulare, Scapania undulata, Jungermannia atrovirens and Cratoneuron filicinum. The associated vascular flora is fairly consistent, contributing about one third of species richness of the community. Nevertheless, vascular species only occur occasionally, even if their cover is often remarkable (mostly Cardamine amara).

Platyhypnidium riparioides is a typical moss of fastflowing and well oxygenated crenic waters. Because it can be encountered on both limestone and siliceous substrata, it occurs within a variety of submerged crenic communities (Schmidt 1993). Nevertheless, Platyhypnidium riparioides reaches a peak of abundance within the Oxyrrhynchietum rusciformis association, of which it is considered a weak character species (Marstaller 1987). Our 18 relevés can be clearly allocated to this association, the only one within the Platyhypnidion rusciformis alliance, belonging to the order Leptodictyetalia riparii. This order includes the submerged bryophyte communities of springs found within the collinar vegetation belt, irrigated by neutral to alkaline waters (Schmidt 1993).

From the classification dendrogram (Fig. 1a) the Oxyrrhynchietum rusciformis is a fairly heterogeneous vegetation type. The particularly species-poor subcluster ORty (rel. 1-6) corresponds to the typical subassociation. Within the subassociation a variant differentiated by Cratoneuron filicinum can be identified (rel. 5-6). The subcluster ORcc (rel. 7-15) is noticeably less species-poor than the typical subassociation, from which it is clearly differentiated by the prominence of Palustriella commutata and the occurrence of several vascular species. It can be referred to the Oxyrrhynchietum rusciformis subass. cratoneuretosum commutati, first described by Schmidt (1993) from central Germany. Finally, the subcluster ORsu (rel. 16-18), showing clear transitional features to the Scapanietum undulatae, can be assigned to the Oxyrrhynchietum rusciformis subass. scapanietosum undulatae, first typified by Marstaller (1987).

The Oxyrrhynchietum rusciformis is the submerged bryophyte community most commonly found in the study area. Both the Oxyrrhynchietum rusciformis typicum and the subassociation cratoneuretosum commutati are restricted to the collinar vegetation belt, in springs flowing from limestone substrata, with alkaline, base-rich, calcareous and mesotrophic waters. The Oxyrrhynchietum rusciformis scapanietosum undulatae has a very local distribution at a spring within the montane vegetation belt on a porphyritic substratum. Crenic waters are neutral, base-poor and oligotrophic.

\section{Cinclidotetum aquatici (CA - Tab. 3)}

This community (cluster CA) corresponds to an extremely species-poor stand dominated by the water moss Cinclidotus aquaticus, also including Platyhypnidium riparioides as the only other species. Cinclidotus aquaticus is a robust moss with strongly thickened leaf margins, adapted to fast-flowing waters. It has been reported as abundant on submerged carbonate rocks, especially in large karstic systems subjected to summer drought (Reynolds 1998).

The stand can be regarded as a floristically impoverished form of the association Cinclidotetum aquatici, that is placed in the alliance Cinclidotion fontinaloidis and in the order Leptodictyetalia riparii. The alliance includes crenic bryophyte communities occurring on limestone, which are intermittently irrigated in the summer.

A stand of the Cinclidotetum aquatici occurs in the collinar belt of the southern slopes of the Brenta Dolomites, within the Rio Bianco spring, which has a very high discharge flow and alkaline, base-rich, calcareous and nutrient-rich waters.

\section{Brachythecio rivularis-Hygrohypnetum luridi (BH - Tab. 4)}

Cluster BH includes 7 relevés in which the moss Brachythecium rivulare is generally the most abundant and only constant plant, and other bryophytes (mainly Palustriella commutata and Plagiomnium undulatum) can sometimes contribute to the mat. Moreover, the floristic composition of these relevés is markedly enriched by the vascular element, with Cardamine amara being relatively frequent, and Geranium robertianum and Epilobium alsinifolium locally prominent.

Our vegetation relevés have sufficient floristic similarity with the Brachythecio rivularis-Hygrohypnetum luridi to be subsumed within it. The absence of the differential species Hygrohypnum luridum does not negate this syntaxonomical diagnosis, because it also occurs in other phytosociological tables of the associa- 
tion (Marstaller 1987, Schmidt 1993). Nevertheless, the dominant moss Brachythecium rivulare can be regarded no more than a weak character species of the association, due to its broad ecological amplitude (Dierßen 2001). The Brachythecio rivularis-Hygrohypnetum luridi belongs within the alliance Brachythecion rivularis, including the amphibian neutro-basophilous associations colonizing running waters, from the plain to the alpine belts.

As reflected in the classification dendrogram, the Brachythecio rivularis-Hygrohypnetum luridi exhibits a certain degree of floristic heterogeneity. According to Schmidt (1993) relevés 1-4 can be assigned to the typical variant of the association, whereas relevés 5-7 correspond to the subvariant of Rhizomnium punctatum.

The stands of the Brachythecio rivularis-Hygrohypnetum luridi are only intermittently submerged and occur in springs flowing from limestone outcrops (except for rel. 1). Crenic waters are generally alkaline, base-rich, calcareous and oligotrophic. The typical variant was encountered only in the montane vegetation belt, whereas the subvariant of Rhizomnium punctatum seems to be restricted to the high-collinar one.

\section{Phytocoenon of Hygroamblystegium tenax (HT -} Tab. 5)

Cluster HT includes 4 relevés, in which Hygroamblystegium tenax is the only constant species, also occurring as dominant or, in one case, as codominant. Other relatively frequent bryophytes are Brachythecium rivulare and Conocephalum conicum. Vascular species are few in number, concentrated within rel. 3 and not abundant (except Cardamine amara).

From the floristic viewpoint, the community shows clear affinities with the Brachythecio rivularis-Hygrohypnetum luridi, as reflected in the classification dendrogram (Fig. 1a). It is, however, fairly well distinguished from this association, mainly by the constancy of Hygroamblystegium tenax and the occurrence of Conocephalum conicum. Considering that the community seems to be floristically unique, we assigned it to a phytocoenon of Hygroamblystegium tenax. This syntaxonomic choice is only provisional, because this type of vegetation has been under-sampled in the project; further study may characterise it better.

The phytocoenon of Hygroamblystegium tenax is restricted to the collinar vegetation belt. Crenic waters are alkaline, base-rich, calcareous and mesotrophic.

\subsubsection{Non-submerged bryophyte or vascular plant vegetation (Montio-Cardaminetea)}

About two thirds of relevé set (93) include crenic communities only occasionally submerged by flowing waters. Bryophytes are dominant in most stands, forming dense mats from which vascular plants, of minor structural importance, emerge, although some of them can sometimes be abundant. Vascular plants only achieve constant dominance in shaded stands.

All non-submerged crenic communities are grouped together in the comprehensive class Montio-Cardaminetea, with its single order, the Montio-Cardaminetalia (Zechmeister \& Mucina 1994). The largest component of the order is the alliance Cratoneurion commutati, including the most species-rich communities, usually occurring on carbonate substrata. Other alliances represented in the study area are the Cardamino-Montion, including crenic communities on siliceous substrata, with oligotrophic and acidic waters, and the Caricion remotae, whose communities, occurring at oligophotic sites, are dominated by phanerogams.

\section{CRATONEURION COMMUTATI}

The numerical classification of 49 relevés which could readily be incorporated within the Cratoneurion commutati produced a dendrogram, in which six different plant communities can be distinguished (Fig. 1b). Most of them exhibit marked bryophyte dominance. In only two communities do dominant tall herbs form a thick herbaceous layer, whose shading effect inhibits the development of a dense bryophyte carpet.

\section{Cratoneuretum commutati (CC - Tab. 6)}

The community (cluster CC) includes 21 relevés in which the moss, Palustriella commutata, is consistently dominant, often forming mounds or banks. Other bryophytes that contribute to the mat, although typically in a minor way, are Bryum pseudotriquetrum, Plagiomnium undulatum, Palustriella falcata, Brachythecium rivulare and Conocephalum conicum. Vascular plants are numerically predominant compared to bryophytes, but many are only scattered in occurrence and have low cover values. Several species (Crepis paludosa, Deschampsia caespitosa, Petasites albus, Chaerophyllum hirsutum) contribute relatively frequently, occasionally with consistent abundance.

Palustriella commutata is a eurasiatic moss normally found in mineral-rich springs on carbonate substrata, under relatively mild climatic conditions. It is regarded as the only character species of the association Cratoneuretum commutati, to which our stands correspond closely. Relevés 17 to 21 are clearly differentiated by the constancy and significant occurrence of the moss Palustriella falcata. They are to be assigned to a variant of Palustriella falcata, which can be seen as a floristic transition to the following community (Cratoneuretum falcati). The higher species richness of these relevés confirms their ecotonal floristic nature.

The Cratoneuretum commutati is an association occurring on permanently moist ground, irrigated by base-rich, calcareous, oligotrophic to mesotrophic waters. In most cases the association has the character of a flush, with the dominant moss forming a carpet over gently-sloping ground; more rarely it can occur 
over steep surfaces. The Cratoneuretum commutati is the most widespread Cratoneurion commutati-community in the study area, where it spans a wide altitudinal range. The typical form occurs from the upper collinar to the montane vegetation belt, whereas the Palustriella falcata variant is restricted to the upper montane vegetation belt and is irrigated by slightly more oligotrophic waters.

\section{Cratoneuretum falcati (CF - Tab. 7)}

This community, corresponding to the cluster CF, was sampled through 15 relevés. Most of them are characterized by the large predominance of the moss Palustriella falcata, a circumboreal species that can colonise irrigated calcareous rocks, springs and fens (Dierßen 2001). Only two other bryophytes (Palustriella commutata and Bryum pseudotriquetrum) have more than $50 \%$ frequency. Globally, this community is poorer in vascular species than the Cratoneuretum commutati, and vascular plants occur sporadically and with generally low cover. In some stands only Epilobium alsinifolium and Cardamine amara, and Caltha palustris and Carex paniculata (two sites) can attain prominence.

Relevés 1-6, are particularly species-poor and here Palustriella falcata achieves its maximum prevalence. They correspond to a floristically impoverished form of the association Cratoneuretum falcati that is distributed throughout the Alps. Relevés 7-13 are less species-poor and differentiated by the constant and prominent occurrence of Palustriella commutata. They correspond to a variant of Palustriella commutata of the Cratoneuretum falcati and have a clear transitional nature towards the Cratoneuretum commutati. Relevé 14 and 15 are differentiated by the cover peaks of Caltha palustris and Carex paniculata, respectively. They are treated here as further variants of the Cratoneuretum falcati.

The impoverished form of the Cratoneuretum falcati was encountered in springs from the upper montane to the subalpine vegetation belt, whereas the variant of Palustriella commutata seems to be restricted to the montane one. Crenic waters of both types are base-rich and calcareous. Trophic status ranges from (more frequently) oligotrophic in the impoverished form, to (generally) mesotrophic in the variant of Palustriella commutata. The variant of Caltha palustris occurs along the edge of a spring flowing from dolomite rocks; whereas the variant of Carex paniculata was sampled within a sulphurous helocrene.

\section{Phytocoenon of Cratoneuron filicinum (CFi - Tab. 8)}

This community (cluster CFi) comprises 7 somewhat heterogeneous relevés from the floristic viewpoint. Floristically, the lowest common denominator is the occurrence of the moss Cratoneuron filicinum as a constant and dominant species within the bryophyte layer, where Palustriella commutata and Philonotis calcarea are also fairly frequent and abundant. The associated vascular flora includes a majority of sporadical species, with several that can be locally conspicuous (Cardamine amara, Mentha longifolia, Rorippa amphibia, Epilobium parviflorum).

This distinctive vegetation type is syntaxonomically rather isolated. Bryophyte species such as Cratoneuron filicinum, Palustriella commutata and Philonotis calcarea provide a link with the crenic communities of the Cratoneurion commutati, whereas the occurrence of Mentha longifolia, Rorippa amphibia and Epilobium parviflorum suggests some affinity with disturbed habitats within the class Molinio-Arrhenatheretea. In spite of this floristic ambiguity, the community can be placed inside the alliance Cratoneurion commutati, as a phytocoenon of Cratoneuron filicinum. It is impossible to arrange it as a true association, due to the broad ecological amplitude of the dominant moss. Relevés 6 and 7 are distinguished by the exclusive occurrence of Rorippa amphibia, Epilobium parviflorum and Calliergonella cuspidata, differentiating a variant of Rorippa amphibia.

The phytocoenon of Cratoneuron filicinum is a prevalently collinar crenic vegetation, sometimes occurring at anthropogenically disturbed sites. The dominant moss is relatively common at lower altitudes and it seems to be characteristic of somewhat eutrophic waters (Birse 1980; Philippi \& Oberdorfer 1992). In our samples the waters are base-rich, calcareous, generally mesotrophic and, occasionally eutrophic, due to agricultural nitrate input.

\section{Phytocoenon of Palustriella decipiens (PD - Tab. 9)}

Palustriella decipiens is a eurasiatic moss that is preferentially encountered on calcareous micaschists in crenic waters with low mineral content (Dierßen 2001). It must also survive more severe climates than Palustriella commutata. We sampled a crenic stand in which Palustriella decipiens was largely dominant within a floristic assemblage with low bryophyte number and cover and numerically prevalent, and sometimes prominent, vascular plants. Due to the prominence of Palustriella decipiens this unique relevé was located in the alliance Cratoneurion commutati. Too little phytosociological material is available to typify the community at the association level.

The phytocoenon of Palustriella decipiens occurs in a spring in the upper montane belt of the Fassa Dolomites. Crenic waters are relatively base-rich, calcareous and oligotrophic.

\section{Chaerophyllo-Cardaminetum asarifoliae (CCa -} Tab. 10)

This community occurs very rarely in the study area, being recorded from only one site. From the floristic viewpoint, the sampled stand is overwhelmingly dominated by the tall perennial herb, Cardamine asarifolia, 
with Cirsium montanum, Chaerophyllum hirsutum and Petasites albus the most prominent vascular associates. Bryophytes are scarcer than in the other Cratoneurion commutati communities. Only Brachythecium rivulare, Cratoneuron filicinum, Plagiomnium undulatum attain extensive cover.

The most obvious floristic affinities of this stand are with the Chaerophyllo-Cardaminetum asarifoliae. This association was described by Gerdol \& Tomaselli (1988) from the Northern Apennines, where it occurs on acidic sandstone outcrops. In contrast, our stand occurs on lime-rich bedrock, which explains some of the floristic differences with the Apennine stands. For this reason, it is possible to distinguish a calcicolous Petasites albus-variant of the original Chaerophyllo-Cardaminetum asarifoliae.

This variant was encountered at a spring in the montane vegetation belt, irrigated by base-rich, calcareous and mesotrophic waters.

\section{Phytocoenon of Petasites albus (PA -Tab. 11)}

This community corresponds to the PA cluster and was sampled through four relevés. It comprises fragmentary stands united by the dominance of the tall herb Petasites albus. As a group, vascular plants make the most notable contribution to the vegetation cover, with Cirsium montanum, Chaerophyllum hirsutum and
Crepis paludosa the most frequent associates, and Eupatorium cannabinum occasionally prominent. Bryophytes may also contribute to the ground cover, though as a group they are far less frequent and abundant than the vascular plants. Palustriella commutata and Cratoneuron filicinum are the most prominent bryophyte species.

The community is fairly variable in its floristics, although invariably characterized by the dominance of Petasites albus. Because this species has only a weak diagnostic power, the community could not be typified at the association level, but only as a phytocoenon of Petasites albus. This was accommodated within the Crautonerion commutati, based on the frequent occurrence of several species belonging to this alliance.

The phytocoenon of Petasites albus bears some floristic resemblance to the Petasites albus-variant of the Chaerophyllo-Cardaminetum asarifoliae, from which it differs mainly by the impoverished combination of characteristic species and the occurrence of several tall hygrophilous herbs belonging to the alliance Calthion. These floristic differences can be explained; the phytocoenon of Petasites albus generally forms a transition zone around the spring core.

The phytocoenon is restricted to the montane vegetation belt and to lime-rich outcrops. Waters are baserich, calcareous and from oligotrophic to mesotrophic.

Tab. 1. Scapanietum undulatae (SU). (D) = differential species.

\begin{tabular}{|c|c|c|c|c|c|}
\hline $\begin{array}{l}\text { Relevè } \mathrm{n} \text {. } \\
\text { Spring Code } \\
\text { Altitude }(\mathrm{m} \text { a.s.l.) } \\
\text { Inclination }\left({ }^{\circ}\right) \\
\text { Relevé area }\left(\mathrm{m}^{2}\right) \\
\text { Vascular plant cover }(\%) \\
\text { Bryophyte cover }(\%) \\
\text { N. of species }\end{array}$ & $\begin{array}{c}1 \\
\text { AD } 1353 \\
1353 \\
1 \\
1.5 \\
- \\
100 \\
2\end{array}$ & $\begin{array}{c}2 \\
\text { CV1435 } \\
1435 \\
3 \\
1 \\
- \\
90 \\
5\end{array}$ & $\begin{array}{c}3 \\
\text { CV1421 } \\
1421 \\
20 \\
1 \\
15 \\
80 \\
6\end{array}$ & $\begin{array}{c}4 \\
\text { AD } 1353 \\
1353 \\
70 \\
0.4 \\
- \\
90 \\
4\end{array}$ & $\operatorname{Fr}(\%)$ \\
\hline $\begin{array}{l}\text { SCAPANIETUM UNDULATAE } \\
\text { RACOMITRION ACICULARIS and } \\
\text { BRACHYTHECIETALIA PLUMOSI } \\
\text { Scapania undulata }\end{array}$ & 1 & 1 & 2 & 2 & 100 \\
\hline $\begin{array}{l}\text { Subass. FONTINALIETOSUM ANTIPYRETICAE } \\
\text { Fontinalis antipyretica } \text { (D) }\end{array}$ & 5 & 5 & 4 & 1 & 100 \\
\hline $\begin{array}{l}\text { PLAGIOCHILA PORELLOIDES - variant } \\
\text { Plagiochila porelloides (D) }\end{array}$ & . & . & . & 4 & 25 \\
\hline $\begin{array}{l}\text { PLATYHYPNIDIO-FONTINALIETEA ANTIPYRETICAE } \\
\text { Brachythecium rivulare }\end{array}$ & . & + & . & . & 25 \\
\hline $\begin{array}{l}\text { COMPANIONS } \\
\text { MONTIO-CARDAMINETEA } \\
\text { Cardamine amara } \\
\text { Saxifraga stellaris }\end{array}$ & $\cdot$ & . & $\begin{array}{l}1 \\
1\end{array}$ & . & $\begin{array}{l}25 \\
25\end{array}$ \\
\hline $\begin{array}{l}\text { OTHER COMPANIONS } \\
\text { Porella platyphylla } \\
\text { Crepis paludosa } \\
\text { Plagiothecium cavifolium } \\
\text { Rhizomnium magnifolium }\end{array}$ & . & $\begin{array}{l}\dot{.} \\
\dot{+} \\
+\end{array}$ & $\begin{array}{l}\dot{+} \\
+ \\
\dot{.}\end{array}$ & $\begin{array}{l}2 \\
. \\
.\end{array}$ & $\begin{array}{l}25 \\
25 \\
25 \\
25\end{array}$ \\
\hline
\end{tabular}


Tab. 2. Oxyrrhynchietum rusciformis (OR). (D) = differential species.

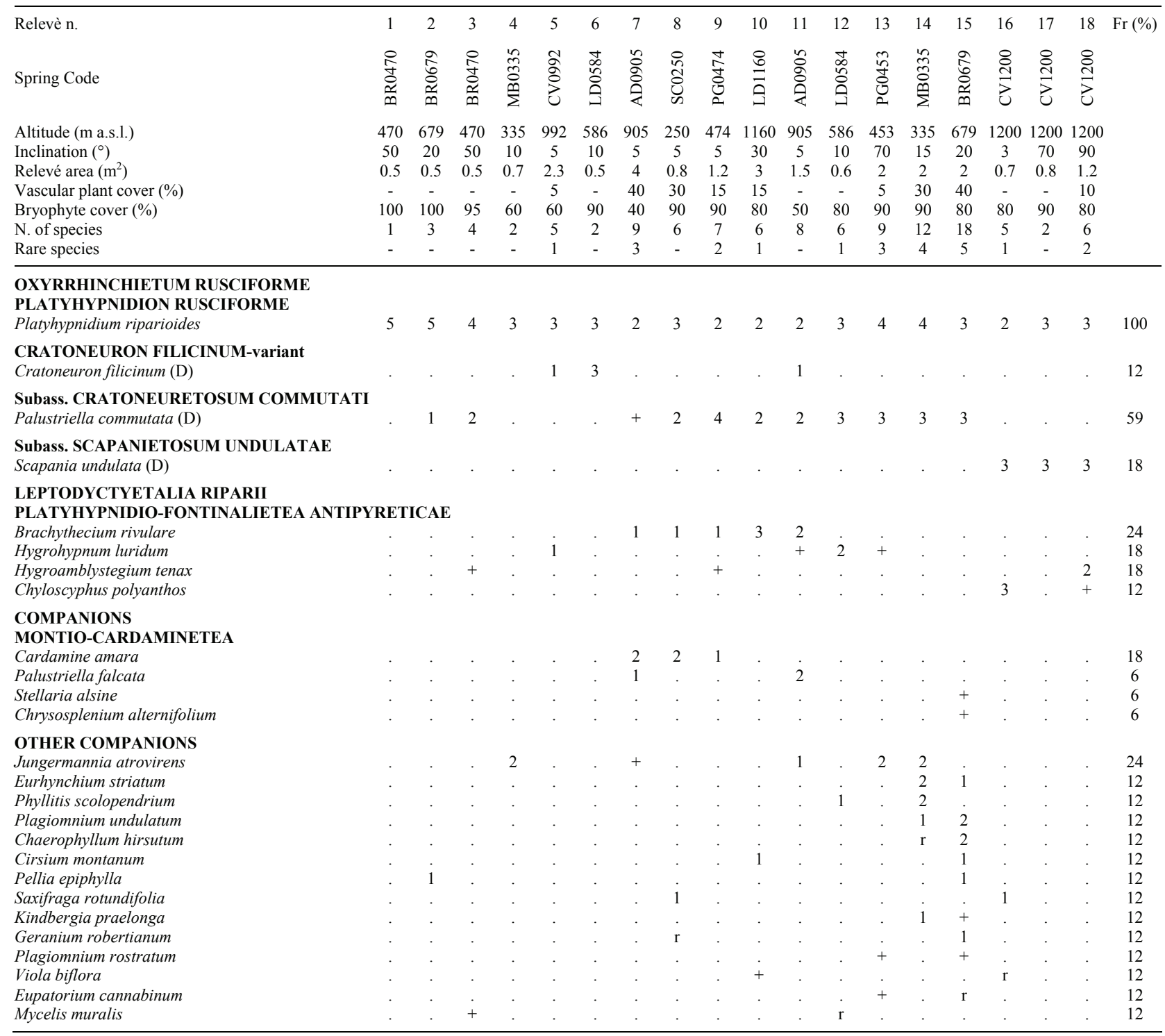

Tab. 3.Cinclidotetum aquatici (CA).

\begin{tabular}{lc}
\hline Relevè n. & 1 \\
Spring Code & BR0686 \\
Altitude (m a.s.1.) & 686 \\
Inclination $\left({ }^{\circ}\right)$ & 5 \\
Relevé area $\left(\mathrm{m}^{2}\right)$ & 0.8 \\
Vascular plant cover (\%) & - \\
Bryophyte cover $(\%)$ & 100 \\
N. of species & 2 \\
\hline CINCLIDOTETUM AQUATICI & \\
CINCLIDOTION FONTINALOIDIS & 5 \\
Cinclidotus aquaticus & \\
LEPTODYCTYETALIA RIPARII & \\
PLATYHYPNIDIO-FONTINALIETEA & \\
ANTIPYRETICAE & \\
Platyhypnidium riparioides & \\
\hline
\end{tabular}


Tab. 4. Brachythecio rivularis-Hygrohypnetum luridi $(\mathrm{BH})$. (D) $=$ differential species.

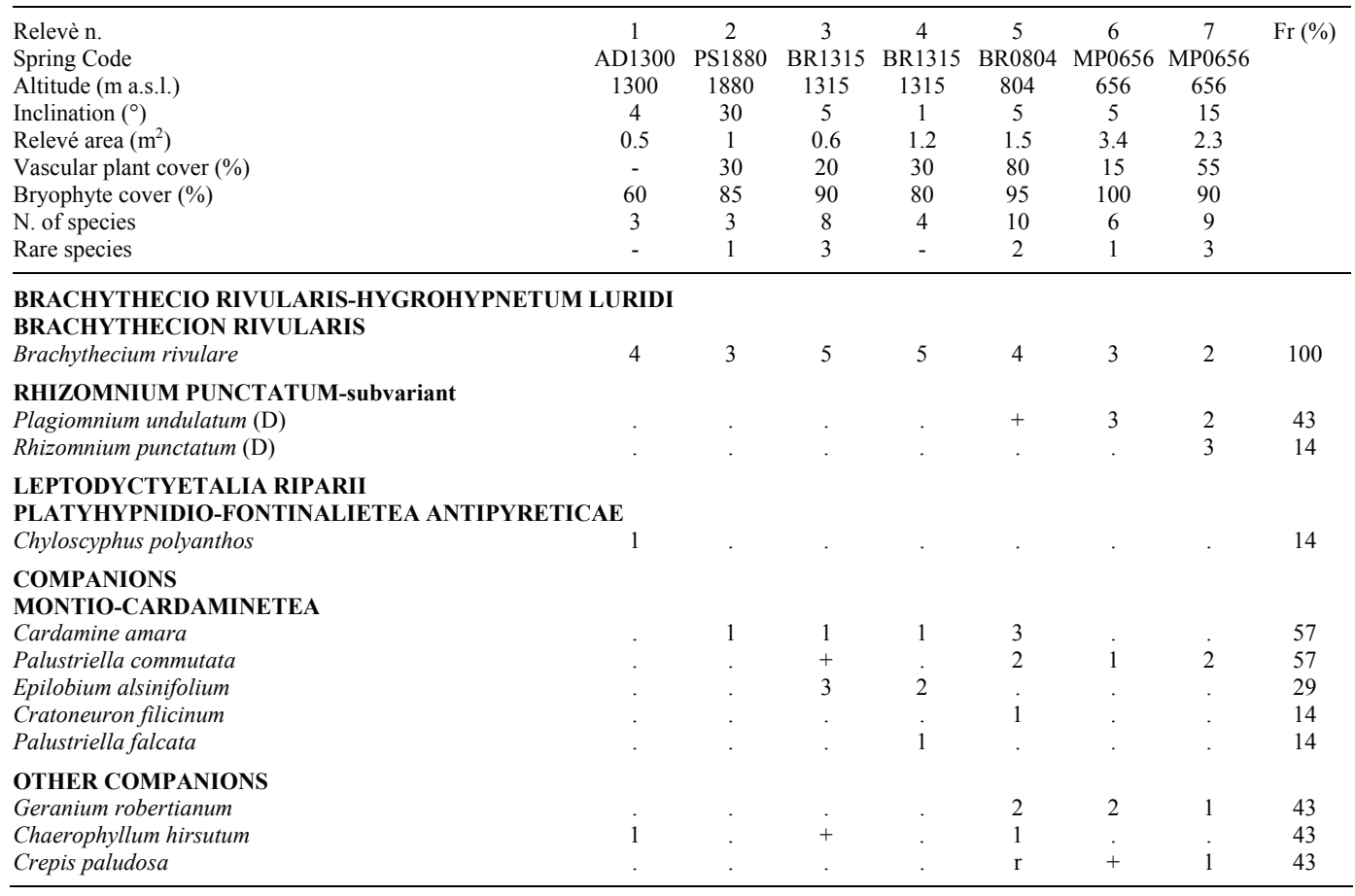

Tab. 5. Phytocoenon of Hygroamblystegium tenax (HT). (D) = differential species.

\begin{tabular}{|c|c|c|c|c|c|}
\hline Relevè n. & 1 & 2 & 3 & 4 & $\operatorname{Fr}(\%)$ \\
\hline Spring Code & BR0470 & BR0470 & CV0250 & $\mathrm{BC} 0170$ & \\
\hline Altitude (m a.s.1.) & 470 & 470 & 250 & 170 & \\
\hline Inclination $\left(^{\circ}\right)$ & 75 & 75 & 5 & 5 & \\
\hline Relevé area (m2) & 0.5 & 0.8 & 3 & 1 & \\
\hline Vascular plant cover $(\%)$ & - & - & 40 & - & \\
\hline Bryophyte cover $(\%)$ & 90 & 95 & 60 & 70 & \\
\hline N. of species & 4 & 6 & 8 & 4 & \\
\hline \multicolumn{6}{|c|}{ Phytocoenon of HYGROAMBLYSTEGIUM TENAX } \\
\hline Hygroamblystegium tenax & 3 & 2 & 3 & 3 & 100 \\
\hline \multicolumn{6}{|c|}{ BRACHYTHECION RIVULARIS } \\
\hline Brachythecium rivulare & . & 3 & 2 & . & 50 \\
\hline Conocephalum conicum (D) & 1 & + & . & . & 50 \\
\hline Cratoneuron filicinum (D) & . & . & . & 1 & 25 \\
\hline \multicolumn{6}{|l|}{ COMPANIONS } \\
\hline Cardamine amara & . & . & 3 & . & 25 \\
\hline Palustriella commutata & . & 2 & . & . & 25 \\
\hline \multicolumn{6}{|l|}{ OTHER COMPANIONS } \\
\hline Didymodon tophaceus & 1 & 1 & . & . & 50 \\
\hline Hygroamblystegium varium & . & . & . & 2 & 25 \\
\hline Jungermannia $s p$. & . & 2 & . & . & 25 \\
\hline Brachythecium rutabulum & . & . & . & 1 & 25 \\
\hline Cirsium montanum & . & . & 1 & . & 25 \\
\hline Epilobium parviflorum & . & . & 1 & . & 25 \\
\hline Equisetum sylvaticum & . & . & 1 & . & 25 \\
\hline Jungermannia atrovirens & 1 & . & . & . & 25 \\
\hline Calystegia sepium & . & . & $\dot{+}$ & . & 25 \\
\hline Viola biflora & . & . & + & . & 25 \\
\hline
\end{tabular}


Tab. 6. Cratoneuretum commutati (CC). (D) = differential species.

\begin{tabular}{|c|c|c|c|c|c|c|c|c|c|c|c|c|c|c|c|c|c|c|c|c|c|c|}
\hline \multirow{2}{*}{$\begin{array}{l}\text { Relevè n. } \\
\qquad \text { Spring Code }\end{array}$} & 1 & 2 & 3 & 4 & 5 & 6 & 7 & 8 & 9 & 10 & 11 & 12 & 13 & 14 & 15 & 16 & 17 & 18 & 19 & 20 & \multirow{2}{*}{\multicolumn{2}{|c|}{$1 \operatorname{Fr}(\%)$}} \\
\hline & $\frac{\hat{\sigma}}{8}$ & $\underset{⿱ 亠 乂}{\stackrel{\Xi}{二}}$ & 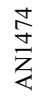 & $\frac{8}{8}$ & 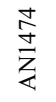 & $\begin{array}{l}\stackrel{2}{\curvearrowright} \\
\hat{0} \\
\stackrel{1}{0}\end{array}$ & 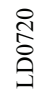 & $\begin{array}{l}n \\
\stackrel{n}{n} \\
\approx\end{array}$ & $\begin{array}{l}\stackrel{0}{n} \\
\text { ò } \\
\frac{0}{m}\end{array}$ & $\begin{array}{l}\stackrel{0}{2} \\
\stackrel{2}{0} \\
\frac{n}{m}\end{array}$ & $\begin{array}{l}\stackrel{n}{2} \\
\text { o } \\
\text { m }\end{array}$ & 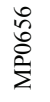 & 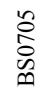 & $\begin{array}{l} \pm \\
n \\
\infty \\
0 \\
0\end{array}$ & હิ & $\begin{array}{l}\text { ô } \\
\text { ڤે̊ } \\
\text { ดे }\end{array}$ & $\begin{array}{l}\stackrel{4}{6} \\
\stackrel{2}{2}\end{array}$ & $\begin{array}{l}\infty \\
\infty \\
\infty \\
\infty \\
\approx\end{array}$ & $\begin{array}{l}\stackrel{\infty}{n} \\
\stackrel{\sim}{\approx} \\
\frac{\sim}{n}\end{array}$ & $\frac{\widetilde{o}}{\stackrel{n}{1}}$ & 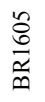 & \\
\hline Altitude (m a.s.1.) & $\stackrel{\circ}{\circ}$ & $\underset{⿱}{ \pm}$ & $\stackrel{ \pm}{ \pm}$ & $\stackrel{-}{\circ}$ & $\underset{⿱}{ \pm}$ & $\stackrel{1}{\circ}$ & $\stackrel{\overbrace{}}{N}$ & $\stackrel{n}{\simeq}$ & $\stackrel{\circ}{n}$ & $\stackrel{\circ}{n}$ & $\stackrel{2}{2}$ & $\stackrel{\wp}{6}$ & $\stackrel{2}{2}$ & $\stackrel{+}{\infty}$ & तิ & ڤ్ & $\stackrel{+}{6}$ & $\underset{\infty}{\infty}$ & $\stackrel{\infty}{\sim}$ & ָ̌ & $\stackrel{n}{0}$ & \\
\hline Inclination $\left({ }^{\circ}\right)$ & 25 & 7 & 7 & 45 & 7 & 30 & - & 5 & 7 & 7 & 7 & 30 & 7 & 3 & 2 & 40 & 10 & 60 & 5 & 85 & 45 & \\
\hline Relevé area $\left(\mathrm{m}^{2}\right)$ & 3.0 & 1.0 & 1.0 & 2.0 & 1.0 & 8.0 & 0.3 & 1.0 & 5.0 & 2.0 & 1.0 & 3.0 & 1.0 & 0.4 & 1.7 & 2.0 & 4.0 & 4 & 0.6 & 1 & 8.0 & \\
\hline Vascular plant cover $(\%)$ & 70 & 70 & 80 & 10 & 80 & 75 & 20 & 25 & 20 & 40 & 60 & 35 & 100 & 20 & 95 & 5 & 60 & 75 & 20 & 35 & 60 & \\
\hline Bryophyte cover $(\%)$ & 70 & 90 & 100 & 30 & 100 & 30 & 100 & 90 & 90 & 70 & 75 & 50 & 95 & 60 & 70 & 20 & 85 & 90 & 50 & 85 & 70 & \\
\hline N. of species & 9 & 5 & 10 & 9 & 7 & 3 & 2 & 3 & 15 & 6 & 5 & 13 & 7 & 12 & 11 & 9 & 22 & 17 & 17 & 13 & 21 & \\
\hline Rare species & 3 & - & - & - & - & 1 & 1 & - & 4 & - & - & 4 & 1 & 4 & 2 & 4 & 3 & 4 & 4 & 6 & 9 & \\
\hline $\begin{array}{l}\text { CRATONEURETUM COMMUTATI } \\
\text { Palustriella commutata }\end{array}$ & 4 & 5 & 5 & 3 & 5 & 5 & 5 & 5 & 5 & 4 & 5 & 3 & 3 & 4 & 4 & 2 & 5 & 4 & 2 & 3 & 4 & 100 \\
\hline $\begin{array}{l}\text { PALUSTRIELLA FALCATA-variant } \\
\text { Palustriella falcata (D) }\end{array}$ & . & $\cdot$ & $\cdot$ & . & . & $\cdot$ & . & . & . & $\cdot$ & . & . & . & . & $\cdot$ & $\cdot$ & 1 & 2 & 1 & 3 & 1 & 24 \\
\hline $\begin{array}{l}\text { CRATONEURION COMMUTATI } \\
\text { Palustriella decipiens } \\
\text { Cratoneuron filicinum } \\
\text { Saxifraga aizoides } \\
\text { Pinguicula alpina } \\
\text { Equisetum variegatum (D) } \\
\text { Philonotis calcarea }\end{array}$ & $\begin{array}{l}\cdot \\
\dot{.} \\
\dot{.} \\
\dot{.}\end{array}$ & $\begin{array}{l}\dot{.} \\
\dot{.} \\
\dot{.} \\
\dot{.}\end{array}$ & $\begin{array}{l}. \\
. \\
. \\
. \\
.\end{array}$ & $\begin{array}{l}+ \\
\cdot \\
\cdot \\
\cdot \\
\cdot \\
\cdot\end{array}$ & $\begin{array}{l}\cdot \\
. \\
. \\
. \\
.\end{array}$ & $\begin{array}{l}\dot{.} \\
\dot{.} \\
\dot{.} \\
\dot{.}\end{array}$ & $\begin{array}{l}. \\
. \\
. \\
.\end{array}$ & $\begin{array}{l}. \\
. \\
. \\
.\end{array}$ & $\begin{array}{l}+ \\
\dot{.} \\
\dot{.} \\
\dot{.} \\
\dot{ } \\
.\end{array}$ & $\begin{array}{l}\cdot \\
\cdot \\
\cdot \\
\cdot \\
\cdot \\
\cdot\end{array}$ & $\begin{array}{l}. \\
. \\
.\end{array}$ & $\begin{array}{l}\dot{+} \\
\dot{.} \\
\dot{.} \\
\dot{.}\end{array}$ & $\begin{array}{l}+ \\
. \\
. \\
. \\
. \\
.\end{array}$ & $\begin{array}{l}. \\
. \\
. \\
.\end{array}$ & $\begin{array}{l}. \\
. \\
. \\
.\end{array}$ & $\begin{array}{l}. \\
. \\
.\end{array}$ & $\begin{array}{l}. \\
2 \\
+ \\
1 \\
1\end{array}$ & $\begin{array}{l}i \\
. \\
.\end{array}$ & $\begin{array}{l}i \\
. \\
. \\
.\end{array}$ & $\begin{array}{l}+ \\
+ \\
+ \\
\cdot \\
\cdot\end{array}$ & $\begin{array}{l}\cdot \\
\dot{+} \\
+ \\
\dot{.} \\
\dot{y}\end{array}$ & $\begin{array}{c}19 \\
14 \\
10 \\
10 \\
5 \\
5\end{array}$ \\
\hline $\begin{array}{l}\text { MONTIO-CARDAMINETALIA and MO } \\
\text { CARDAMINETEA }\end{array}$ & & & & & & & & & & & & & & & & & & & & & & \\
\hline Brachythecium rivulare (D) & . & . & . & . & 1 & . & . & . & . & 1 & . & 1 & . & . & . & . & . & + & 1 & . & . & 24 \\
\hline Epilobium alsinifolium & . & . & 2 & . & 3 & . & . & . & . & $\cdot$ & . & . & . & . & . & . & 1 & 2 & . & . & . & 19 \\
\hline Cardamine amara & . & . & 1 & . & . & . & . & . & . & . & . & . & . & . & . & . & 1 & 3 & . & . & . & 14 \\
\hline Saxifraga stellaris & . & . & . & . & . & . & . & . & . & . & . & . & . & . & . & . & 2 & + & . & . & . & 10 \\
\hline Chyloscyphus pallescens & . & $\cdot$ & . & . & . & . & . & . & . & . & . & . & . & . & + & . & . & . & . & . & . & 5 \\
\hline $\begin{array}{l}\text { COMPANIONS } \\
\text { ADIANTETEA }\end{array}$ & & & & & & & & & & & & & & & & & & & & & & \\
\hline Pellia endiviifolia (D) & . & . & . & . & . & . & . & . & + & . & . & . & . & . & . & . & . & . & . & + & . & 10 \\
\hline Hymenostylium recurvirostre & . & . & . & . & . & . & . & . & . & . & . & . & . & . & . & . & . & . & . & 1 & . & 5 \\
\hline Hymenostylium recurvirostre var. latifolium & $\cdot$ & $\cdot$ & . & . & . & . & . & . & . & $\cdot$ & . & . & . & . & . & . & . & . & . & $\cdot$ & + & 5 \\
\hline OTHER COMPANIONS & & & & & & & & & & & & & & & & & & & & & & \\
\hline Crepis paludosa & 1 & 2 & 1 & 1 & 1 & 1 & . & . & . & . & 2 & . & 3 & . & . & . & 1 & . & 1 & + & 1 & 57 \\
\hline Deschampsia caespitosa & 3 & 3 & 1 & . & 1 & . & . & 1 & + & . & . & . & . & 1 & 3 & . & + & . & . & . & . & 43 \\
\hline Petasites albus & 1 & 1 & 2 & 1 & 1 & . & . & . & 1 & 1 & . & . & . & . & . & . & 1 & . & 1 & . & . & 43 \\
\hline Chaerophyllum hirsutum & . & . & . & . & . & . & . & . & 1 & 2 & 3 & 1 & . & . & . & . & 2 & + & 2 & 2 & . & 38 \\
\hline Bryum pseudotriquetrum & 1 & . & + & . & . & . & . & . & . & . & . & . & . & . & + & . & + & 1 & + & 1 & 1 & 38 \\
\hline Plagiomnium undulatum & . & . & . & + & . & . & . & . & 1 & 2 & + & 1 & 3 & . & 1 & . & . & . & . & . & . & 33 \\
\hline Viola biflora & + & . & + & . & . & . & . & . & . & . & . & . & . & . & . & + & + & + & . & 1 & 1 & 33 \\
\hline Adenostyles glabra & . & 1 & 1 & . & . & . & . & . & . & . & . & . & . & . & . & . & 1 & + & . & . & 2 & 24 \\
\hline Conocephalum conicum & . & . & . & . & . & . & . & . & 1 & 1 & . & . & . & . & . & 2 & . & . & 1 & + & . & 24 \\
\hline Cirsium montanum & 1 & . & . & 2 & 3 & . & . & . & . & . & . & . & . & . & . & . & . & . & . & . & 1 & 19 \\
\hline Geranium robertianum & . & . & . & . & . & . & . & . & $\mathrm{r}$ & . & 1 & 2 & . & . & . & + & . & . & . & . & . & 19 \\
\hline Equisetum sylvaticum & $\cdot$ & . & . & . & . & . & . & 2 & 1 & $\cdot$ & . & . & : & 1 & . & . & . & . & $\dot{2}$ & . & . & 14 \\
\hline Plagiomnium elatum & . & . & . & . & . & . & . & . & . & . & . & . & 1 & . & + & . & . & . & 2 & . & . & 14 \\
\hline Eupatorium cannabinum & . & . & . & . & . & . & . & . & . & . & . & . & . & + & 3 & . & . & . & . & . & . & 10 \\
\hline Agrostis stolonifera & . & . & . & . & . & . & . & . & 1 & . & . & . & . & . & . & . & . & . & 1 & . & . & 10 \\
\hline Carex flacca & . & . & 1 & . & . & . & . & . & . & . & . & . & . & . & . & . & 1 & . & . & . & . & 10 \\
\hline Saxifraga rotundifolia & . & . & . & . & . & . & . & . & . & . & . & . & . & . & . & . & . & . & 1 & 1 & . & 10 \\
\hline Aneura pinguis & 1 & . & . & . & . & . & . & . & . & . & . & . & . & . & + & . & . & . & . & . & . & 10 \\
\hline Calliergonella cuspidata & . & . & . & . & . & . & . & . & . & . & . & . & . & + & 1 & . & . & . & . & . & . & 10 \\
\hline Polygonum viviparum & . & . & . & . & . & . & . & . & . & . & . & . & . & . & . & . & . & + & . & . & 1 & 10 \\
\hline Sesleria caerulea & . & . & . & . & . & . & . & . & . & . & . & . & . & . & . & . & . & . & . & + & 1 & 10 \\
\hline Tofieldia calyculata & . & . & . & . & . & . & . & . & . & . & . & . & . & . & . & . & + & . & . & . & 1 & 10 \\
\hline Arabis alpina & . & . & . & . & . & . & . & . & . & . & . & . & . & . & . & . & + & . & . & + & . & 10 \\
\hline Oxyrrhynchium schleicheri & . & . & . & . & . & . & . & . & . & . & . & + & . & . & . & . & . & . & + & . & . & 10 \\
\hline Filipendula ulmaria & . & . & . & + & . & . & . & . & . & . & . & . & . & + & . & . & . & . & . & . & . & 10 \\
\hline Stellaria nemorum & . & . & . & . & . & . & . & . & . & . & . & . & . & + & . & . & . & + & . & . & . & 10 \\
\hline Brachypodium sylvaticum & . & . & . & . & . & . & . & . & . & . & . & + & $\mathrm{r}$ & . & . & . & . & . & . & . & . & 10 \\
\hline Mycelis muralis & . & . & . & . & . & . & . & . & . & . & . & $\mathrm{r}$ & . & . & . & + & . & . & . & . & . & 10 \\
\hline
\end{tabular}


Table 7. Cratoneuretum falcati $(\mathrm{CF})$. (D) = differential species.

\begin{tabular}{|c|c|c|c|c|c|c|c|c|c|c|c|c|c|c|c|c|}
\hline Relevè n. & 1 & 2 & 3 & 4 & 5 & 6 & 7 & 8 & 9 & 10 & 11 & 12 & 13 & 14 & 15 & $\operatorname{Fr}(\%)$ \\
\hline Spring Code & 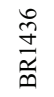 & $\begin{array}{l}\frac{n}{2} \\
\frac{\sqrt{n}}{n}\end{array}$ & 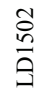 & $\underset{\sim}{\stackrel{\Xi}{\Xi}}$ & 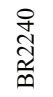 & $\begin{array}{l}\stackrel{+}{ \pm} \\
\underset{\sim}{\simeq}\end{array}$ & $\frac{8}{\stackrel{8}{+}}$ & $\frac{a}{\stackrel{n}{a}}$ & $\begin{array}{l}\stackrel{D}{Ð} \\
\stackrel{\sim}{\sim}\end{array}$ & $\begin{array}{l}\frac{a}{n} \\
\frac{n}{n}\end{array}$ & $\frac{n}{\frac{n}{2}}$ & 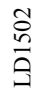 & $\begin{array}{l}\infty \\
\infty \\
\infty \\
\approx\end{array}$ & $\underset{\varpi}{\stackrel{\Xi}{J}}$ & $\begin{array}{l}n \\
\stackrel{n}{n} \\
\tilde{n}\end{array}$ & \\
\hline Altitude (m a.s.1.) & $\stackrel{\circ}{\stackrel{一}{ \pm}}$ & $\frac{n}{2}$ & $\stackrel{\Im}{\mathscr{n}}$ & $\stackrel{さ}{I}$ & 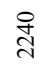 & $\stackrel{\stackrel{+}{ \pm}}{\mathrm{N}}$ & \& & $\stackrel{a}{2}$ & 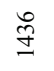 & $\stackrel{2}{m}$ & $\approx$ & 응 & 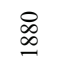 & $\stackrel{\mathbb{I}}{=}$ & $\stackrel{n}{n}$ & \\
\hline Inclination $\left(^{\circ}\right)$ & 90 & 60 & 85 & 2 & 30 & 20 & 7 & 30 & 30 & 15 & 15 & 10 & 20 & 2 & 10 & \\
\hline Relevé area $\left(\mathrm{m}^{2}\right)$ & 1 & 0.7 & 0.5 & 1 & 0.7 & 1.5 & 3.0 & 0.3 & 0.8 & 0.6 & 3 & 1 & 1 & 1.5 & 2 & \\
\hline Vascular plant cover $(\%)$ & - & - & - & 5 & 5 & 5 & - & 25 & 80 & 25 & 10 & - & 60 & 80 & 95 & \\
\hline Bryophyte cover $(\%)$ & 100 & 100 & 98 & 95 & 95 & 70 & 10 & 70 & 90 & 100 & 90 & 80 & 100 & 95 & 20 & \\
\hline N. of species & 5 & 1 & 1 & 5 & 8 & 3 & 9 & 13 & 5 & 3 & 15 & 9 & 8 & 6 & 9 & \\
\hline Rare species & 2 & - & - & - & 2 & - & 3 & 5 & 1 & - & 6 & 1 & 1 & 1 & 1 & \\
\hline \multicolumn{17}{|l|}{ CRATONEURETUM FALCATI } \\
\hline Palustriella falcata & 5 & 5 & 5 & 5 & 5 & 4 & 1 & 2 & 3 & 4 & 5 & 3 & 5 & 5 & 2 & 100 \\
\hline Polygonum viviparum (D) & . & . & . & . & + & . & . & . & . & . & . & . & 1 & . & . & 13 \\
\hline \multicolumn{9}{|l|}{ PALUSTRIELLA COMMUTATA-variant } & 2 & 3 & 2 & 2 & 1 & . & 2 & 53 \\
\hline \multicolumn{17}{|l|}{ CALTHA PALUSTRIS-variant } \\
\hline Caltha palustris (D) & . & . & . & . & . & . & . & . & . & . & . & . & . & 4 & . & 7 \\
\hline \multicolumn{17}{|l|}{ CAREX PANICULATA-variant } \\
\hline \multicolumn{17}{|l|}{ CRATONEURION COMMUTATI } \\
\hline Cratoneuron filicinum & . & . & . & 1 & 1 & . & + & . & . & . & + & + & . & . & . & 33 \\
\hline Palustriella decipiens & . & . & . & . & . & . & . & 1 & . & . & . & 2 & . & . & . & 13 \\
\hline Saxifraga aizoides & . & . & . & . & . & . & . & . & . & . & . & . & 3 & . & . & 7 \\
\hline Arabis subcoriacea & . & . & . & 1 & . & . & . & . & . & . & . & . & . & . & . & 7 \\
\hline \multicolumn{17}{|c|}{ MONTIO-CARDAMINETALIA and MONTIO-CARDAMINETEA } \\
\hline Brachythecium rivulare (D) & . & . & . & . & . & 2 & . & . & . & . & 1 & 2 & . & . & . & 20 \\
\hline Epilobium alsinifolium & . & . & . & . & . & . & . & . & . & 2 & 1 & . & . & 2 & . & 20 \\
\hline Cardamine amara & . & . & . & 1 & . & . & . & . & . & . & . & . & 1 & 2 & . & 20 \\
\hline Silene pusilla & . & . & . & . & . & . & . & 2 & . & . & . & . & . & . & . & 7 \\
\hline Saxifraga stellaris & . & . & . & . & 1 & . & . & . & . & . & . & . & . & . & . & 7 \\
\hline \multicolumn{17}{|l|}{ COMPANIONS } \\
\hline \multicolumn{17}{|l|}{ SCHEUCHZERIO-CARICETEA NIGRAE } \\
\hline Aneura pinguis & . & . & . & . & . & . & . & + & . & . & + & . & . & . & . & 13 \\
\hline Campylium stellatum & . & . & . & . & . & . & . & . & . & . & . & + & . & . & $\mathrm{r}$ & 13 \\
\hline Juncus alpino-articulatus & . & . & . & . & . & . & . & . & . & . & . & . & . & . & 3 & 7 \\
\hline Scorpidium revolvens & . & . & . & . & . & . & . & . & . & . & . & . & . & . & 1 & 7 \\
\hline Tofieldia calyculata & . & . & . & . & . & . & . & . & . & . & . & . & . & . & 1 & 7 \\
\hline Carex frigida & . & . & . & . & . & . & . & . & . & . & + & . & . & . & . & 7 \\
\hline Selaginella selaginoides & . & . & . & . & . & . & . & $\mathrm{r}$ & . & . & . & . & . & . & . & 7 \\
\hline \multicolumn{17}{|l|}{ OTHER COMPANIONS } \\
\hline Bryum pseudotriquetrum & . & . & . & . & + & 1 & . & 2 & 2 & . & 2 & 1 & . & + & 1 & 53 \\
\hline Jungermannia sp. & 1 & . & . & . & . & . & + & . & 1 & . & . & . & . & . & . & 20 \\
\hline Jungermannia atrovirens & . & . & . & . & . & . & + & . & . & . & . & 1 & . & . & . & 13 \\
\hline Poa alpina & . & . & . & . & $\dot{+}$ & . & . & . & . & . & . & . & 1 & . & . & 13 \\
\hline Viola biflora & . & . & . & $\dot{+}$ & . & . & . & . & . & . & . & . & 1 & . & . & 13 \\
\hline Hymenostylium recurvirostre var. latifolium & + & . & . & . & . & . & . & . & . & . & $\dot{+}$ & . & . & . & . & 13 \\
\hline Lophozia ventricosa & . & . & . & . & . & . & + & + & . & . & . & . & . & . & . & 13 \\
\hline
\end{tabular}


Tab. 8. Phytocoenon of Cratoneuron filicinum (CFi). (D) = differential species.

\begin{tabular}{|c|c|c|c|c|c|c|c|c|}
\hline Relevè n. & 1 & 2 & 3 & 4 & 5 & 6 & 7 & $\operatorname{Fr}(\%)$ \\
\hline Spring Code & AN0590 & BR1379 & AN0430 & AN0590 & CS1350 & ВC0565 & ВC0565 & \\
\hline Altitude (m a.s.1.) & 590 & 1379 & 430 & 590 & 1350 & 565 & 565 & \\
\hline Inclination $\left({ }^{\circ}\right)$ & 15 & 5 & 1 & 15 & 35 & 7 & 5 & \\
\hline Relevé area $\left(\mathrm{m}^{2}\right)$ & 2.5 & 0.3 & 1 & 2.7 & 2.3 & 1.5 & 1.5 & \\
\hline Vascular plant cover $(\%)$ & 40 & 5 & 80 & 40 & 100 & 30 & 30 & \\
\hline Bryophyte cover (\%) & 90 & 90 & 30 & 90 & 10 & 45 & 40 & \\
\hline N. of species & 7 & 10 & 7 & 8 & 8 & 14 & 5 & \\
\hline Rare species & 2 & 5 & 3 & 2 & 3 & 7 & - & \\
\hline \multicolumn{9}{|c|}{ Phytocoenon of CRATONEURON FILICINUM } \\
\hline \multicolumn{9}{|c|}{ RORIPPA AMPHIBIA-variant } \\
\hline Rorippa amphibia (D) & . & . & . & . & . & 1 & 3 & 29 \\
\hline Epilobium parviflorum (D) & . & . & . & . & . & 2 & 1 & 29 \\
\hline Calliergonella cuspidata (D) & . & . & . & . & . & 1 & 1 & 29 \\
\hline \multicolumn{9}{|c|}{ CRATONEURION COMMUTATI } \\
\hline Palustriella commutata & 1 & 3 & 2 & 1 & . & & & 57 \\
\hline Philonotis calcarea & . & 2 & . & . & . & 1 & 1 & 43 \\
\hline \multicolumn{9}{|c|}{ MONTIO-CARDAMINETALIA and MONTIO-CARDAMINETEA } \\
\hline Cardamine amara & $\mathrm{r}$ & . & 5 & 3 & 3 & . & . & 57 \\
\hline Brachythecium rivulare (D) & . & . & + & . & 1 & . & . & 29 \\
\hline Philonotis seriata & . & 2 & . & . & . & . & . & 14 \\
\hline Philonotis fontana & . & . & . & . & . & + & . & 14 \\
\hline \multicolumn{9}{|l|}{ COMPANIONS } \\
\hline \multicolumn{9}{|c|}{ MOLINIO-ARRHENATHERETEA } \\
\hline Mentha longifolia & . & . & . & 2 & 4 & . & . & 29 \\
\hline Equisetum palustre & $\mathrm{r}$ & . & . & 1 & . & . & . & 29 \\
\hline Mentha aquatica & 4 & . & . & . & . & . & . & 14 \\
\hline Agrostis stolonifera & . & . & . & . & . & 1 & . & 14 \\
\hline Ranunculus repens & 1 & . & . & . & . & . & . & 14 \\
\hline Potentilla reptans & . & . & . & . & . & + & . & 14 \\
\hline \multicolumn{9}{|l|}{ OTHER COMPANIONS } \\
\hline Bryum pseudotriquetrum & 2 & 1 & . & 1 & . & . & . & 29 \\
\hline Equisetum arvense & . & . & . & . & $\dot{r}$ & $\dot{+}$ & . & 29 \\
\hline
\end{tabular}

Tab. 9. Phytocoenon of Palustriella decipiens (PD).

\begin{tabular}{lc}
\hline Relevè n. & 1 \\
Spring Code & MD1670 \\
Altitude (m a.s.1.) & 1670 \\
Inclination $\left({ }^{\circ}\right)$ & 5 \\
Relevé area $\left(\mathrm{m}^{2}\right)$ & 1 \\
Vascular plant cover (\%) & 40 \\
Bryophyte cover (\%) & 70 \\
N. of species & 15 \\
\hline Phytocoenon of PALUSTRIELLA DECIPIENS & \\
CRATONEURION COMMUTATI & \\
Palustriella decipiens & 4 \\
MONTIO-CARDAMINETALIA and MONTIO- & \\
CARDAMINETEA & \\
Cardamine amara & 2 \\
Epilobium alsinifolium & + \\
COMPANIONS & \\
Tussilago farfara & \\
Poa alpina & \\
Rhizomnium punctatum & \\
Viola biflora & \\
Alchemilla lineata & \\
Aster bellidiastrum & \\
Bryum pseudotriquetrum & \\
Campylium stellatum & \\
Deschampsia caespitosa & \\
Geum rivale & Petasites albus \\
Cerastium fontanum & 1 \\
\hline & 1 \\
\hline & + \\
\hline
\end{tabular}


Tab. 10. Chaerophyllo-Cardaminetum asarifoliae $(\mathrm{CCa})$. (D) $=$ differential species.

\begin{tabular}{lc}
\hline Relevè n. & 1 \\
Spring Code & AD1235 \\
Altitude (m a.s.1.) & 1235 \\
Inclination ( ${ }^{\circ}$ & 45 \\
Relevé area (m2) & 8 \\
Vascular plant cover (\%) & 80 \\
Bryophyte cover (\%) & 30 \\
N. of species & 13 \\
\hline CHAEROPHYLLO-CARDAMINETUM ASARIFOLIAE & \\
Cardamine asarifolia & \\
Chaerophyllum hirsutum (D) & 4 \\
PETASITES ALBUS-variant & 2 \\
Petasites albus (D) & \\
CRATONEURION COMMUTATI & 2 \\
Cratoneuron filicinum & \\
MONTIO-CARDAMINETALIA and MONTIO- & 2 \\
CARDAMINETEA & \\
Brachythecium rivulare (D) & \\
COMPANIONS & \\
Cirsium montanum & \\
Plagiomnium undulatum & \\
Platyhypnidium riparioides & \\
Saxifraga rotundifolia & Bryum pseudotriquetrum \\
Geranium robertianum & \\
Plagiomnium rostratum & \\
Conocephalum conicum & 3 \\
\hline
\end{tabular}

Tab. 11. Phytocoenon of Petasites albus (PA). (D) = differential species.

\begin{tabular}{|c|c|c|c|c|c|}
\hline Relevè n. & 1 & 2 & 3 & 4 & $\operatorname{Fr}(\%)$ \\
\hline Spring Code & AT1052 & AD0905 & BR0658 & BS1527 & \\
\hline Altitude (m a.s.1.) & 1052 & 905 & 658 & 1527 & \\
\hline Inclination $\left(^{\circ}\right)$ & 3 & 5 & 20 & 10 & \\
\hline Relevé area (m2) & 1 & 2 & 5.4 & 1.7 & \\
\hline Vascular plant cover $(\%)$ & 40 & 95 & 85 & 50 & \\
\hline Bryophyte cover $(\%)$ & 5 & 40 & 20 & 40 & \\
\hline N. of species & 7 & 23 & 13 & 14 & \\
\hline Rare species & 2 & 10 & 8 & 5 & \\
\hline \multicolumn{6}{|c|}{ Phytocoenon of PETASITES ALBUS } \\
\hline Petasites albus (D) & 2 & 3 & 4 & 2 & 100 \\
\hline Cirsium montanum (D) & 1 & 2 & . & + & 75 \\
\hline \multicolumn{6}{|c|}{ CRATONEURION COMMUTATI } \\
\hline Palustriella commutata & 1 & . & 2 & . & 50 \\
\hline Cratoneuron filicinum & . & 1 & . & 2 & 50 \\
\hline Saxifraga aizoides & . & 1 & . & . & 25 \\
\hline Palustriella decipiens & . & . & . & + & 25 \\
\hline \multicolumn{6}{|c|}{ MONTIO-CARDAMINETALIA and MONTIO-CARDAMINETEA } \\
\hline Brachythecium rivulare (D) & . & 2 & . & . & 25 \\
\hline Epilobium alsinifolium & . & . & . & 2 & 25 \\
\hline Pohlia wahlenbergii & . & . & + & . & 25 \\
\hline Cardamine amara & $\mathrm{r}$ & . & . & . & 25 \\
\hline \multicolumn{6}{|l|}{ COMPANIONS } \\
\hline Chaerophyllum hirsutum & 1 & 1 & . & + & 75 \\
\hline Crepis paludosa & . & 1 & . & 2 & 50 \\
\hline Mentha longifolia & . & 1 & . & . & 25 \\
\hline Geum rivale & . & . & . & + & 25 \\
\hline \multicolumn{6}{|l|}{ ADIANTION } \\
\hline Conocephalum conicum & . & + & 1 & . & 50 \\
\hline Pellia endiviifolia & . & 1 & + & . & 50 \\
\hline \multicolumn{6}{|l|}{ OTHER COMPANIONS } \\
\hline Plagiomnium elatum & . & 1 & . & + & 50 \\
\hline
\end{tabular}




\section{CARDAMINO-MONTION}

Another 30 phytosociological relevés were crudely separated from the original data set and assigned to the alliance Cardamino-Montion based on a preliminary analysis of their floristic composition. The numerical classification of these relevés produced a dendrogram (Fig. 1c), in which three different plant communities can clearly be distinguished.

\section{Montio-Bryetum schleicheri (MB - Tab. 12)}

This community was sampled through 18 relevés and it is labelled as MB in the classification dendrogram. The physiognomy of the stands is rather variable, because bryophytes and vascular plants vary considerably in their cover and variety. The moss carpet attains dominance in the majority of stands, even if there are quite frequent stands in which the vascular element prevails.

Among the bryophytes, Brachythecium rivulare is the only constant and sometimes dominant species. Bryum pseudotriquetrum is the only other moss that is frequent throughout. Scapania undulata, Philonotis seriata, P. fontana and Sanionia uncinata are less consistent through the community as a whole, but locally prominent.

The vascular flora is rather varied in its composition and cover. The only constant species is Saxifraga stellaris, whose scattered rosettes are very characteristic. Other herbs that are frequent throughout are Deschampsia caespitosa, Cardamine amara and Epilobium alsinifolium, while Stellaria nemorum and Viola biflora are more occasional. Among the rarer vascular plants, Stellaria alsine attains dominance at one stand.

The classification of this community raises particular difficulties because it is troublesome to characterise it with diagnostic species. Neither the dominant moss (Brachythecium rivulare), nor the dominant vascular plant (Saxifraga stellaris) can be used to typify an association, because their coenological amplitudes are too large. There are also general floristic and habitat similarities between our community and the MontioBryetum schleicheri association, which forms the most widespread and common Cardamino-Montion association in the subalpine and alpine belts of the Alps. Nevertheless, phytosociologically it is difficult to place our relevés in the typical variant of this association, because its character species (Bryum schleicheri) is absent from all our stands. One solution would be to consider Epilobium alsinifolium as a territorial characteristic species of the Montio-Bryetum schleicheri, because this species is centred in this community within the study area.

The high occurrence of Brachythecium rivulare and Cardamine amara is helpful for assigning our relevés to a variant of the Montio-Bryetum schleicheri, differentiated by these two species. This variant was reported from the Swiss Alps by Geissler (1976), where it colonises subalpine and alpine springs with fast-flowing waters. Relevés 17 and 18, in which Stellaria alsine becomes an important component, are to be attributed to another specific variant.

In Trentino the Brachythecium rivulare-Cardamine amara variant of the Montio-Bryetum schleicheri is mostly widespread from the high-montane to the subalpine vegetation belt of the siliceous massifs. Springs are generally typical helocrenes with circumneutral, basepoor and oligotrophic waters. The Stellaria alsine-variant was encountered at a spring in the montane vegetation belt of the Lagorai range with slightly more alkaline, less base-poor waters.

\section{Solenostomo-Hygrohypnetum smithii (SH - \\ Tab. 13)}

This community includes 4 relevés, labelled $\mathrm{SH}$ in the dendrogram, in which the alpine moss Hygrohypnum smithii is the only constant species. The associated flora is a rather species-poor assemblage, with rare, and especially sparse, vascular plants. Bryophytes largely prevail numerically, but none of them rivals Hygrohypmnum smithii in abundance and frequency. Some of them (Jungermannia exsertifolia subsp. cordifolia, Sciuro-hypnum latifolium, Sanionia uncinata) are locally prominent. The most abundant vascular species is Saxifraga stellaris.

The community has close floristic affinities with the alpine association, Solenostomo-Hygrohypnetum smithii, described by Geissler (1976), sharing the two character species (Hygrohypnum smithii and Jungermannia exsertifolia subsp. cordifolia). Relevé 1 corresponds to the typical variant of the Solenostomo-Hygrohypnetum smithii and relevé 2 , with only two species, to an extremely impoverished form of the association. Relevés 3 and 4 belong to a chionophilous Anthelia juratzkanavariant, also differentiated by Marsupella brevissima, Pohlia obtusifolia, P. drummondii. Other species, such as Cephalozia ambigua, Kiaeria starkei, Luzula alpinopilosa, Marsupella boeckii, Salix herbacea, provide a further link between this variant and the chionophilous communities of the Salicetea herbaceae.

The Solenostomo-Hygrohypnetum smithii is restricted to the alpine vegetation belt of the siliceous massifs, where spring-heads are generally associated with late snow-beds, flowing from the debris cover of some rock-glaciers. Waters are cold, subneutral, basepoor and oligotrophic.

\section{Blindio-Scapanietum undulatae (BS - Tab. 14)}

This community was sampled with 8 relevés, labelled as BS in the classification dendrogram (Fig. 1c). It has a quite distinct general character, but shows a certain range of compositional variation, more particularly in the proportion of its different structural components, bryophytes and vascular plants. However, bryophytes, prevail numerically and as cover in the majority of stands. The constants of the community are very few, 
only Scapania undulata (bryophytes) and Saxifraga stellaris (vascular plants). Among the bryophytes, Warnstorfia exannulata attains high frequency and abundance also Philonotis seriata is fairly common. Of the vascular plants, only Deschampsia caespitosa is also quite frequent and abundant throughout.

Our relevés show a close floristic resemblance with the association Blindio-Scapanietum undulatae to which they can be assigned. The linkage between our stands and this association is given by the constant occurrence of Scapania undulata (treated here as a differential species), the presence of Blindia acuta (the only true character species of the association) and the occurrence of other bryophytes belonging to the specific character combination of the association (Philonotis seriata and Marsupella emarginata). The occurrence of many mire species among the companions provides further support to the syntaxonomical attribution. The first six relevés of table 14 fit the typical form of the association well; whereas relevés 7 and 8 , differentiated by the leafy hepatics Cephalozia bicuspidata and Gymnocolea inflata and by the occurrence of some chionophilous species of the class Salicetea herbaceae, can be referred to a specific Cephalozia bicuspidata-variant.

The Blindio-Scapanietum undulatae occurs from the upper montane to the subalpine vegetation belt of siliceous massifs. Crenic waters are base- and nutrient-poor with $\mathrm{pH}$ values ranging from 5.9 to 7.0 (average value 6.2). The Cephalozia bicuspidata-variant is restricted to the subalpine belt and is irrigated by slightly more acidic and oligotrophic waters.

\section{CARICION REMOTAE}

A further set of 14 relevés was extracted from the original data set and attributed to the alliance Caricion remotae. The relevé set is homogeneous and can therefore be referred to a unique vegetation type.

\section{Cardamino-Chrysosplenietum alternifolii $(\mathrm{CCh}$ - Tab. 15)}

This community is characterized by the general predominance of vascular plants in frequency and cover, with usually subordinate bryophytes. However, the bryophyte flora is fairly rich, but variable in species number and cover. On occasions it makes a substantial contribution to the ground layer, although the frequent species are very few.

No vascular species is constant. Chaerophyllum hirsutum is most frequent and often fairly abundant, while Cardamine amara is less frequent, but more abundant, also dominant or codominant in several stands. Petasites albus attains $50 \%$ frequency and is generally fairly abundant and sometimes prominent. Other vascular plants occurring with some frequency throughout are Oxalis acetosella, which is concentrated in some stands, Chrysosplenium alternifolium, Saxifraga rotundifolia, Stellaria nemorum, Crepis paludosa and Geranium robertianum. Finally, the sedge Carex remota is no more than occasional, although this community is important for it.

Among the bryophytes, Brachythecium rivulare is constant, but only rarely conspicuous, Rhizomnium punctatum is frequent, dominant or subdominant in several stands, and Plagiomnium undulatum is common and fairly abundant.

The affinities of our relevés are clearly with the Cardamino-Chrysosplenietum alternifolii, an association occurring on the mountains of Central Europe, where it colonises montane springs in shaded situations. Chrysosplenium alternifolium is the only character species of this association, in which it is generally constant and dominant (Zechmeister 1993). Nevertheless, in their general floristics, our stands differ significantly from the typical form of the Cardamino-Chrysosplenietum alternifolii. In particular two features distinguish our community. Firstly, both Chrysosplenium alternifolium and the moss Plagiomnium affine are much reduced, the first largely replaced by Chaerophyllum hirsutum. Secondly, some stands are clearly differentiated by the high occurrence of Oxalis acetosella and the moss Sanionia uncinata. The relevés 1-10, where Chaerophyllum hirsutum is constant and fairly abundant, can be referred to the Cardamino-Chrysosplenietum alternifolii subass. chaerophylletosum hirsuti Zechmeister et Steiner 1993. In the study area the subassociation is also differentiated by Petasites albus and Crepis paludosa. Having Oxalis acetosella as a constant and subdominant element relevés 11-14 should be assigned to a new subassociation, Cardamino-Chrysosplenietum alternifolii subass. oxalidetosum acetosellae Tomaselli, Spitale et Petraglia subass. nova hoc loco; table 15, nomenclatural type: relevé 14 (holotypus). Lamium galeobdolon subsp. flavidum, Urtica dioica and the moss Sanionia uncinata are additional differential species of the subassociation, to the above quoted Oxalis acetosella. Another striking, distinctive feature of the subassociation is the extensive cover of the moss layer, that can exceed that of vascular plants. The subassociation is labelled as CChoa in the subsequent figures.

Both subassociations of the Cardamino-Chrysosplenietum alternifolii occur in the montane vegetation belt at oligophotic sites, where shade is provided by trees or tall herbs. Crenic waters are circumneutral, relatively base- and nutrient-poor. High nitrogen content was reported in the Cardamino-Chrysosplenietum alternifolii oxalidetosum acetosellae. This is presumably a consequence of the more peripheral position of this community with respect to the spring-head determining water flow through soils that are richer in organic nutrients.

\subsubsection{Chasmophytic bryophyte-rich communities}

This small subset only includes four relevés occurring in typical rheocrenes on lime-rich bedrocks. The relevés are distributed between two clearly distinct 
communities, both belonging to the alliance Adiantion capilli-veneris. In our opinion, this alliance must be placed in the independent order Adiantetalia capilliveneris and the class Adiantetea, according to the traditional syntaxonomical approach (Braun-Blanquet et al. 1952). This approach has recently been followed for the Mediterranean area, the principal centre of distribution and diversity of the Adiantetea-vegetation (Deil 1996; Privitera \& Puglisi 2004).

Zechmeister's (1993) and Zechmeister \& Mucina's (1994) alternative placing of this alliance within the class Montio-Cardaminetea cannot be maintained, because there is only one character species of this class (Palustriella commutata) occurring with high frequencies in part of these vegetational communities (see the synoptic table of the Adiantion in Zechmeister \& Mucina 1994).

\section{Eucladietum verticillati (EV - Tab. 16)}

This community, sampled through 3 phytosociological relevés, is typically dominated by an extensive mat of bryophytes. Among them, Eucladium verticillatum and Palustriella commutata are constant and quite prominent. Other bryophytes, of which Hymenostylium recurvirostre is frequent and abundant, characteristically play a subordinate part in the ground cover. Pellia endiviifolia, Bryum pseudotriquetrum and Jungermannia atrovirens are more occasional through the community as a whole. The only vascular plant (Pinguicula alpina) has a negligible cover.

The closest floristic affinities of this community are with the association Eucladietum verticillati, occurring from the Mediterranean to central Europe. The Eucladietum verticillati was encountered in some rheocrenes flowing from calcareous rock-faces. In this habitat mosses and algae build calcareous deposits by removing $\mathrm{CO}_{2}$ from the water (Wilmanns 1989). The community is restricted to the collinar vegetation belt; crenic waters are base-rich, calcareous and usually mesotrophic.

\section{Phytocoenon of Pellia endiviifolia (PE - Tab. 17)}

This community was sampled in a hygropetric, almost vertical, rheocrene spring, occurring in the montane belt of the Brenta massif. Like the Eucladietum verticillati, this is essentially bryophytic vegetation, floristically easily defined by the dominance of the thalloid hepatic, Pellia endiviifolia, probably favoured by the locally shaded condition of the vertical rock face. The vascular element is only represented by Adenostyles glabra and Sesleria caerulea and attains low cover.

The community seems to be floristically sufficiently distinct to be recognised. Nonetheless, new data must be added to improve the classification beyond the level of a phytocoenon named from the dominant species and allocated to the alliance Adiantion.
The crenic waters are base-rich, calcareous and oligotrophic.

\subsubsection{Hygrophilous communities}

This very small subset of 3 relevés comprises all the vegetation types recorded in typical helocrenes which are clearly fragments of mire communities and, therefore, to be placed in the class Scheuchzerio-Caricetea nigrae.

\section{Phytocoenon of Eriophorum angustifolium (EA - Tab. 18)}

Eriophorum angustifolium generally forms speciespoor communities in shallow pools within nutrient-poor mires, and along the shores of mountain small lakes (Gerdol \& Tomaselli 1997). Small stands dominated by the common cotton-grass can occasionally also be encountered at a distance from springheads, where water-flow is less vigorous and gentle slopes allow more diffuse irrigation. Our relevés were taken in such areas, within stands where Eriophorum angustifolium dominated a floristic assemblage in which character species of different syntaxa belonging to the class Scheuchzerio-Caricetea nigrae largely prevailed. Our syntaxomic treatment of the community is in accordance with Dierßen $(1982,1996)$ and Gerdol \& Tomaselli (1997).

In the study area the phytocoenon of Eriophorum angustifolium is restricted to the lower subalpine belt of siliceous massifs and is irrigated by circumneutral, basepoor, oligotrophic to mesotrophic waters.

\section{Caricetum nigrae (CN - Tab. 19)}

The Caricetum nigrae is the most widespread fen association in the South-Eastern Alps, where it has been reported from a number of sites (Gerdol 1994; Gerdol \& Tomaselli 1997). Its occurrence within springs is very occasional and restricted to sites where the conditions (gentle sloping, reduced flow) allow a small mire to be formed. The our unique relevé is well characterized by its floristics, including two character species of the association Caricetum nigrae (Carex canescens and Juncus filiformis), and a core of species of the class Scheuchzerio-Caricetea nigrae. The occurrence of some species of the classes Montio-Cardaminetea and Platyhypnidio-Fontinalietea antipyreticae as companions emphasises the floristic relationships of the community with the crenic habitat.

The Caricetum nigrae-stand was sampled in the montane belt of the siliceous Presanella-massif. Waters are subneutral, base-poor and mesotrophic.

\subsubsection{Tall herb communities}

Within this last small subset, composed of only 5 relevés, we grouped all the residual vegetation types having floristic assemblages dominated by tall herbs. 
Nevertheless, the resulting group is rather heterogeneous, including types of plant communities belonging to different phytosociological classes. However, these communities are not particularly relevant to our analysis, because they are confined to the peripheral areas of the springs, and generally represent a floristic transition between the crenic habitat and the surrounding vegetation. Such stands are generally discrete, but form part of a fragmented zonation. This, combined with their marginal position and ecotonal nature, also posed classificatory problems. In only one case was it possible to refer our relevé to an already-known association. In all other cases classification was only provisional due to the scarcity of data.

\section{Phytocoenon of Mentha aquatica (MA - Tab. 20)}

This community is composed of a heterogeneous stand, in which a number of tall herbs form an intricate vegetational mosaic with a dense mat of the pleustophyte, Lemna minor. Among the herbs, Mentha aquatica is largely prevalent, and the bulk of the assemblage comprises species diagnostic of Calthion, Molinietalia and Molinio-Arrhenatheretea. This spring is the only limnocrene in our dataset. It is characterized by largely lentic waters, forming a large pool, 50 and 90 $\mathrm{cm}$ deep. The limnocrene lies in the collinar vegetation belt. Crenic waters are base-rich, calcareous and oligotrophic.

\section{Phytocoenon of Eupatorium cannabinum (EC - Tab. 21)}

This community is characterized floristically by the dominance of the tall herb, Eupatorium cannabinum, and by the occurrence of a set of diagnostic species of the order Convolvuletalia sepium and the class GalioUrticetea. The assemblage includes also some bryophytes (Palustriella decipiens and Plagiomnium rostratum).

The phytocoenon of Eupatorium cannabinum was encountered at the edge of a spring lying at about $800 \mathrm{~m}$ a.s.l., with base- and electrolyte-rich, calcareous and oligotrophic water. The spring is somewhat disturbed by the presence of cement walls to which metal meshes are anchored to prevent rock falls.

\section{Chaerophyllo-Petasitetum officinalis ( $\mathrm{CP}-$ Tab. 22)}

The most important diagnostic species of this community is Petasites hybridus, whose large leaves confer the stand's typical, distinctive physiognomy. The dominant species is Mentha longifolia. Among the vascular plants, Petasites hybridus and Cardamine amara are abundant and subdominant, and the bryophytes are represented by the mosses, Cratoneuron filicinum and Brachythecium rivulare.

The community corresponds most obviously to the association Chaerophyllo-Petasitetum officinalis, typical of stream and river banks from the collinar to the montane belt. The association is placed in the alliance Petasition officinalis (order Convolvuletalia sepium and class Galio-Urticetea).

In the study area, the association was sampled at the edge of a spring in the montane vegetation belt, lying close to meadows that are only occasionally cut. Crenic waters are base- and electrolyte-rich, calcareous and mesotrophic.

\section{Phytocoenon of Mentha longifolia (ML - Tab. 23)}

This community, sampled through 2 relevés, also has Mentha longifolia as a dominant species. The assemblage is, however, quite different from that of the Chaerophyllo-Petasitetum officinalis. Here the floristic bulk is contributed by tall herbs that occur preferentially at sites with moist and nitrogen-rich soils within the montane and subalpine belts. From the phytosociological viewpoint these species are diagnostic of the alliance Rumicion alpini (order Rumicetalia alpini, class Mulgedio-Aconitetea). For this reason a new phytocoenon must be established, with the name of the dominant species, and placed in this new syntaxonomic context.

The Mentha longifolia phytocoenon was sampled at a spring in the montane vegetation belt, close to a cattle shelter within a pasture. It is probably occasionally disturbed by cattle trampling. The spring has a limited discharge, with base-rich, calcareous and eutrophic waters. 
Tab. 12. Montio-Bryetum schleicheri $(\mathrm{MB})$. $(\mathrm{D})=$ differential species; $(\mathrm{TC})=$ territorial character species.

\begin{tabular}{|c|c|c|c|c|c|c|c|c|c|c|c|c|c|c|c|c|c|c|c|}
\hline Relevè n. & 1 & 2 & 3 & 4 & 5 & 6 & 7 & 8 & 9 & 10 & 11 & 12 & 13 & 14 & 15 & 16 & 17 & 18 & $\operatorname{Fr}(\%)$ \\
\hline Spring Code & $\frac{\vec{J}}{\vec{J}}$ & $\frac{n}{n}$ & $\begin{array}{l}n \\
0 \\
0 \\
己\end{array}$ & $\begin{array}{l}n \\
\infty \\
0 \\
z \\
z \\
z\end{array}$ & $\frac{\stackrel{\circ}{2}}{\dot{z}}$ & $\frac{8}{3}$ & $\overrightarrow{\vec{\sigma}}$ & $\underset{\Xi}{\vec{\Xi}}$ & 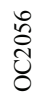 & $\frac{n}{3}$ & 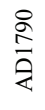 & ¿̊ & $\frac{\stackrel{\Omega}{2}}{\stackrel{2}{4}}$ & 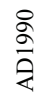 & $\frac{\mathbb{Z}}{\stackrel{8}{2}}$ & 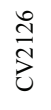 & $\frac{\tilde{d}}{2}$ & 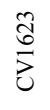 & \\
\hline Altitude (m a.s.1.) & $\vec{\Im}$ & $\stackrel{n}{n}$ & 芯 & $\begin{array}{l}\mathscr{\infty} \\
\infty \\
0\end{array}$ & $\stackrel{\circ}{2}$ & \& & $\bar{\sigma}$ & $\bar{a}$ & $\stackrel{\circ}{\stackrel{N}{~}}$ & $\stackrel{n}{\beth}$ & 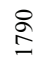 & $\ddot{\stackrel{\sim}{\infty}}$ & $\stackrel{2}{\Omega}$ & $\stackrel{2}{\circ}$ & $\underset{2}{J}$ & 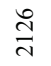 & $\tilde{\widehat{\sigma}}$ & త్ర్త & \\
\hline Inclination $\left(^{\circ}\right)$ & 5 & 5 & 25 & 15 & 10 & 2 & 3 & 7 & 15 & 35 & 5 & 7 & 30 & 40 & 1 & 20 & 3 & 5 & \\
\hline Relevé area $\left(\mathrm{m}^{2}\right)$ & 1 & 2.5 & 0.7 & 2.5 & 4 & 1.5 & 1 & 5 & 4 & 1 & 2 & 0.5 & 4 & 2 & 0.5 & 0.5 & 0.7 & 2 & \\
\hline Vascular plant cover (\%) & 20 & 95 & 30 & 75 & 90 & 70 & 25 & 80 & 70 & 80 & 95 & 30 & 70 & 60 & 40 & 30 & 70 & 100 & \\
\hline Bryophyte cover (\%) & 25 & 95 & 75 & 90 & 60 & 20 & 80 & 50 & 95 & 95 & 15 & 25 & 85 & 50 & 90 & 80 & 70 & 10 & \\
\hline N. of species & 6 & 12 & 18 & 26 & 28 & 14 & 6 & 12 & 20 & 17 & 10 & 9 & 10 & 10 & 8 & 18 & 13 & 16 & \\
\hline Rare species & - & 1 & 2 & 4 & 2 & 3 & - & 2 & 6 & 5 & - & 1 & - & 2 & - & 2 & 3 & 6 & \\
\hline
\end{tabular}

MONTIO-BRYETUM SCHLEICHERI

Epilobium alsinifolium (TC)

BRACHYTHECIUM RIVULARE-CARDAMINE AMARA-variant

Brachythecium rivulare

Cardamine amara

$\begin{array}{lllllllllllllllllll}2 & 5 & 1 & 3 & 1 & . & . & . & 3 & 2 & 2 & 1 & 4 & 2 & 2 & + & + & 1 & 83 \\ 2 & 5 & 3 & 1 & 4 & 3 & 1 & 2 & + & + & . & . & . & . & . & 1 & 2 & 2 & 72\end{array}$

STELLARIA ALSINE-variant

Stellaria alsine (D)

CARDAMINO-MONTENION

Luzula alpinopilosa (D)

Carex frigida (D)

CARDAMINO-MONTION

Saxifraga stellaris

Scapania undulata (D)

Philonotis seriata

Epilobium anagallidifolium

Warnstorfia exannulata (D)

Jungermannia exsertifolia subsp. cordifolia

Warnstorfia sarmentosa (D)

MONTIO-CARDAMINETALIA and MONTIO-CARDAMINETEA

Philonotis fontana

Palustriella decipiens

Chrysosplenium alternifolium

Chyloscyphus pallescens

Palustriella falcata

Philonotis calcarea

Cratoneuron filicinum

Dichodontium palustre

Pohlia wahlenbergii

COMPANIONS

SCHEUCHZERIO-CARICETEA NIGRAE

Carex nigra

Oncophorus virens

Campylium stellatum

Aneura pinguis

Carex canescens

Carex panicea

Pinguicula vulgaris

Sphagnum palustre

Straminergon stramineum

Cardamine rivularis

OTHER COMPANIONS

Deschampsia caespitosa

Bryum pseudotriquetrum

Stellaria nemorum

Rhizomnium punctatum

Viola biflora

Sanionia uncinata

Rhizomnium pseudopunctatum

Peucedanum ostruthium

Alchemilla lineata

Plagiomnium affine

Chyloscyphus polyanthos

Pellia epiphylla

Oxalis acetosella

Saxifraga rotundifolia

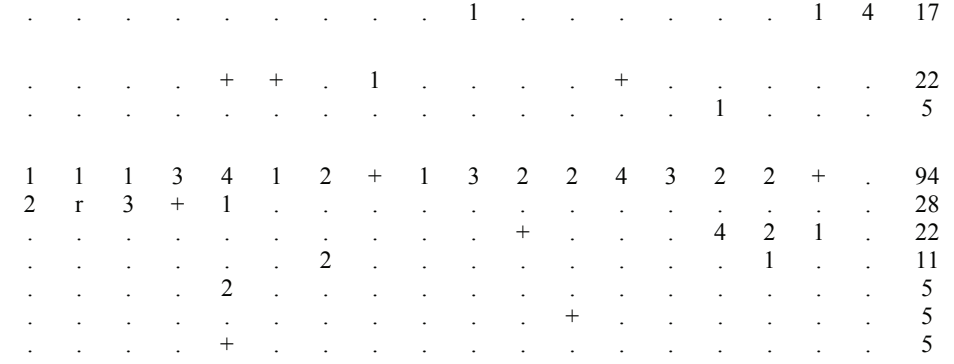


Tab. 12. Continuation.

\begin{tabular}{|c|c|c|c|c|c|c|c|c|c|c|c|c|c|c|c|c|c|c|c|}
\hline Relevè n. & 1 & 2 & 3 & 4 & 5 & 6 & 7 & 8 & 9 & 10 & 11 & 12 & 13 & 14 & 15 & 16 & 17 & 18 & $\operatorname{Fr}(\%)$ \\
\hline Spring Code & $\underset{⿱ 乛 士}{\vec{J}}$ & $\stackrel{n}{\frac{n}{n}}$ & $\begin{array}{l}n \\
\frac{n}{2} \\
j \\
3\end{array}$ & \begin{tabular}{l}
$n$ \\
$\infty$ \\
\hdashline \\
$Z$ \\
$Z$
\end{tabular} & 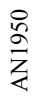 & 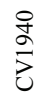 & $\stackrel{\vec{\Xi}}{\vec{\Xi}}$ & $\stackrel{\Xi}{\vec{\Xi}}$ & 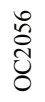 & $\stackrel{n}{\stackrel{n}{J}}$ & 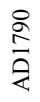 & $\begin{array}{l}n \\
\stackrel{\infty}{\infty} \\
\stackrel{\sim}{>}\end{array}$ & $\frac{\curvearrowright}{\stackrel{2}{2}}$ & $\frac{8}{\stackrel{\Omega}{2}}$ & $\frac{⿱}{2}$ & 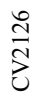 & 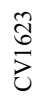 & 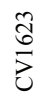 & \\
\hline Altitude (m a.s.1.) & $\overrightarrow{\mathcal{I}}$ & $\stackrel{n}{n}$ & $\sqrt[n]{n}$ & $\sqrt[n]{\infty}$ & $\stackrel{\circ}{2}$ & 올 & $\bar{\Xi}$ & $\bar{a}$ & 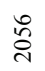 & $\frac{n}{\simeq}$ & § & $\begin{array}{l}n \\
\infty \\
\infty\end{array}$ & $\stackrel{2}{\check{\Xi}}$ & § & $\underset{g}{g}$ & 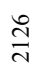 & త్రి & $\tilde{\widehat{\sigma}}$ & \\
\hline Inclination $\left({ }^{\circ}\right)$ & 5 & 5 & 25 & 15 & 10 & 2 & 3 & 7 & 15 & 35 & 5 & 7 & 30 & 40 & 1 & 20 & 3 & 5 & \\
\hline Relevé area $\left(\mathrm{m}^{2}\right)$ & 1 & 2.5 & 0.7 & 2.5 & 4 & 1.5 & 1 & 5 & 4 & 1 & 2 & 0.5 & 4 & 2 & 0.5 & 0.5 & 0.7 & 2 & \\
\hline Vascular plant cover $(\%)$ & 20 & 95 & 30 & 75 & 90 & 70 & 25 & 80 & 70 & 80 & 95 & 30 & 70 & 60 & 40 & 30 & 70 & 100 & \\
\hline Bryophyte cover $(\%)$ & 25 & 95 & 75 & 90 & 60 & 20 & 80 & 50 & 95 & 95 & 15 & 25 & 85 & 50 & 90 & 80 & 70 & 10 & \\
\hline N. of species & 6 & 12 & 18 & 26 & 28 & 14 & 6 & 12 & 20 & 17 & 10 & 9 & 10 & 10 & 8 & 18 & 13 & 16 & \\
\hline Rare species & - & 1 & 2 & 4 & 2 & 3 & - & 2 & 6 & 5 & - & 1 & - & 2 & - & 2 & 3 & 6 & \\
\hline Plagiochila porelloides & . & . & + & . & . & . & . & . & + & . & . & . & . & . & . & + & r & . & 17 \\
\hline Chaerophyllum hirsutum & . & . & $\mathrm{r}$ & $\mathrm{r}$ & . & . & . & . & . & . & + & . & . & . & . & . & . & . & 17 \\
\hline Climacium dendroides & . & . & . & . & . & . & . & . & . & + & . & . & . & . & . & . & 1 & . & 11 \\
\hline Crepis paludosa & . & . & + & . & . & . & . & . & . & . & . & . & . & . & . & . & . & + & 11 \\
\hline Dicranum scoparium & . & . & + & . & + & . & . & . & . & . & . & . & . & . & . & . & . & . & 11 \\
\hline Luzula sudetica & . & . & . & . & + & . & . & . & . & . & . & . & . & . & . & . & . & . & 11 \\
\hline Plagiomnium undulatum & + & + & . & . & . & . & . & . & . & . & . & . & . & . & . & . & . & . & 11 \\
\hline Rhizomnium magnifolium & . & + & . & . & + & . & . & . & . & . & . & . & . & . & . & . & . & . & 11 \\
\hline Ranunculus montanus aggr. & . & . & . & r & + & . & . & . & . & . & . & . & . & . & . & . & . & . & 11 \\
\hline Aconitum napellus & . & . & . & . & $\mathrm{r}$ & . & . & . & . & . & . & . & . & . & . & $\mathrm{r}$ & . & . & 11 \\
\hline
\end{tabular}

Tab. 13. Solenostomo-Hygrohypnetum smithii $(\mathrm{SH})$. $(\mathrm{D})=$ differential species.

\begin{tabular}{|c|c|c|c|c|c|}
\hline Relevè n. & 1 & 2 & 3 & 4 & $\operatorname{Fr}(\%)$ \\
\hline Spring Code & CA2153 & AD2739 & AD2314 & AD2314 & \\
\hline Altitude (m a.s.1.) & 2153 & 2739 & 2314 & 2314 & \\
\hline Inclination $\left({ }^{\circ}\right)$ & 30 & 15 & 3 & 10 & \\
\hline Relevé area $\left(\mathrm{m}^{2}\right)$ & 0.8 & 0.5 & 1 & 7 & \\
\hline Vascular plant cover $(\%)$ & 25 & - & - & 2 & \\
\hline Bryophyte cover $(\%)$ & 80 & 2 & 20 & 10 & \\
\hline N. of species & 13 & 2 & 15 & 11 & \\
\hline Rare species & 8 & 1 & 5 & 3 & \\
\hline \multicolumn{6}{|c|}{ SOLENOSTOMO-HYGROHYPNETUM SMITHII } \\
\hline Hygrohypnum smithii & 5 & 1 & 1 & 2 & 100 \\
\hline Jungermannia exsertifolia subsp. cordifolia & 2 & . & . & . & 25 \\
\hline \multicolumn{6}{|l|}{ ANTHELIA JURATZKANA-variant } \\
\hline Anthelia juratzkana (D) & . & . & 1 & 1 & 50 \\
\hline Marsupella brevissima (D) & . & . & + & 1 & 50 \\
\hline Lophozia opacifolia (D) & . & . & . & 1 & 25 \\
\hline Pohlia obtusifolia (D) & . & . & 1 & . & 25 \\
\hline \multicolumn{6}{|l|}{ CARDAMINO-MONTENION } \\
\hline Luzula alpinopilosa (D) & . & . & . & + & 25 \\
\hline \multicolumn{6}{|l|}{ CARDAMINO-MONTION } \\
\hline Saxifraga stellaris & 3 & . & . & . & 25 \\
\hline Philonotis seriata & 2 & . & . & . & 25 \\
\hline Scapania undulata (D) & 1 & & . & . & 25 \\
\hline \multicolumn{6}{|c|}{ MONTIO-CARDAMINETALIA and MONTIO-CARDAMINETEA } \\
\hline Pohlia wahlenbergii & . & & 1 & . & 25 \\
\hline Philonotis tomentella & . & . & . & + & 25 \\
\hline \multicolumn{6}{|l|}{ COMPANIONS } \\
\hline \multicolumn{6}{|l|}{ SALICETEA HERBACEAE } \\
\hline Pohlia drummondi & . & . & 1 & . & 25 \\
\hline Cephalozia ambigua & . & . & + & & 25 \\
\hline Kiaeria starkei & . & . & + & . & 25 \\
\hline Marsupella boeckii & . & . & + & . & 25 \\
\hline Salix herbacea & . & . & . & + & 25 \\
\hline \multicolumn{6}{|l|}{ OTHER COMPANIONS } \\
\hline Sciuro-hypnum latifolium & . & . & 2 & & 25 \\
\hline Sanionia uncinata & . & . & . & 2 & 25 \\
\hline
\end{tabular}


Tab. 14. Blindio-Scapanietum undulatae (BS). (D) = differential species.

\begin{tabular}{|c|c|c|c|c|c|c|c|c|c|}
\hline Relevè n. & 1 & 2 & 3 & 4 & 5 & 6 & 7 & 8 & $\operatorname{Fr}(\%)$ \\
\hline Spring Code & AD1853 & AD1853 & AD1944 & CA1642 & CV2051(2) & CA1642 & AD2153 & AD2153 & \\
\hline Altitude (m a.s.1.) & 1853 & 1853 & 1944 & 1642 & 2051 & 1642 & 2153 & 2153 & \\
\hline Inclination $\left({ }^{\circ}\right)$ & 5 & 30 & 8 & 5 & 15 & 5 & 5 & 10 & \\
\hline Relevé area (m2) & 4.8 & 0.5 & 2.5 & 1.2 & 2.5 & 1 & 1.6 & 4 & \\
\hline Vascular plant cover $(\%)$ & 15 & 20 & 55 & 65 & 40 & 90 & 80 & 15 & \\
\hline Bryophyte cover $(\%)$ & 50 & 80 & 80 & 40 & 50 & 40 & 25 & 65 & \\
\hline N. of species & 22 & 5 & 14 & 8 & 14 & 17 & 17 & 9 & \\
\hline Rare species & 8 & - & 1 & - & 2 & 6 & 5 & - & \\
\hline \multicolumn{10}{|l|}{ BLINDIO-SCAPANIETUM UNDULATAE } \\
\hline Scapania undulata (D) & 3 & 3 & 1 & 3 & 1 & 3 & 2 & 5 & 100 \\
\hline Blindia acuta & + & . & . & . & . & . & . & 1 & 25 \\
\hline \multicolumn{10}{|l|}{ CEPHALOZIA BICUSPIDATA-variant } \\
\hline Cephalozia bicuspidata (D) & + & . & . & . & . & . & 1 & 1 & 25 \\
\hline Gymnocolea inflata (D) & . & . & . & . & . & . & 1 & 1 & 25 \\
\hline \multicolumn{10}{|l|}{ CARDAMINO-MONTENION } \\
\hline Carex frigida (D) & . & . & + & . & . & . & $\mathrm{r}$ & . & 25 \\
\hline Luzula alpinopilosa (D) & . & . & . & . & . & . & 2 & . & 13 \\
\hline \multicolumn{10}{|l|}{ CARDAMINO-MONTION } \\
\hline Warnstorfia exannulata (D) & 2 & 3 & 2 & 1 & 3 & 2 & . & . & 75 \\
\hline Philonotis seriata & + & . & 2 & 2 & . & 1 & . & . & 50 \\
\hline Marsupella emarginata & 2 & 2 & . & 1 & . & . & . & . & 50 \\
\hline Jungermannia exsertifolia subsp. cordifolia & . & . & . & 1 & . & . & . & . & 13 \\
\hline Scapania paludosa & . & . & . & . & . & 1 & . & . & 13 \\
\hline Viola palustris (D) & . & . & . & . & + & . & . & . & 13 \\
\hline \multicolumn{10}{|c|}{ MONTIO-CARDAMINETALIA and MONTIO-CARDAMINETEA } \\
\hline Saxifraga stellaris & 2 & 2 & 2 & 4 & 2 & 2 & 2 & $\mathrm{r}$ & 100 \\
\hline Dichodontium palustre & + & . & 1 & . & + & . & . & . & 38 \\
\hline Philonotis fontana & . & . & . & . & + & . & . & 1 & 25 \\
\hline Cardamine amara & . & . & . & . & . & 1 & . & . & 13 \\
\hline Chyloscyphus pallescens & . & . & + & . & . & . & . & . & 13 \\
\hline \multicolumn{10}{|l|}{ COMPANIONS } \\
\hline \multicolumn{10}{|l|}{ SCHEUCHZERIO-CARICETEA NIGRAE } \\
\hline Eriophorum angustifolium & . & . & + & . & + & 2 & . & . & 38 \\
\hline Carex echinata & . & . & + & . & 1 & . & . & . & 25 \\
\hline Sphagnum subsecundum & $\dot{+}$ & . & . & . & 1 & . & . & . & 25 \\
\hline Straminergon stramineum & + & . & . & . & . & 1 & . & . & 25 \\
\hline Campylium stellatum & . & . & + & . & . & . & . & + & 25 \\
\hline Carex nigra & . & . & 1 & . & . & . & . & . & 13 \\
\hline Scorpidium revolvens & . & . & 1 & . & . & . & . & . & 13 \\
\hline Sphagnum cuspidatum & . & . & . & . & 1 & . & . & . & 13 \\
\hline Juncus filiformis & . & . & . & . & + & . & . & . & 13 \\
\hline Warnstorfia fluitans & . & . & . & . & . & . & . & + & 13 \\
\hline Parnassia palustris & . & . & . & . & . & . & $\mathrm{r}$ & . & 13 \\
\hline \multicolumn{10}{|l|}{ SALICETEA HERBACEAE } \\
\hline Soldanella pusilla & . & . & . & . & . & 1 & . & . & 13 \\
\hline Anthelia juratzkana & . & . & . & . & . & . & . & $\dot{+}$ & 13 \\
\hline Veronica alpina & . & . & . & . & . & . & $\mathrm{r}$ & . & 13 \\
\hline \multicolumn{10}{|l|}{ OXYCOCCO-SPHAGNETEA } \\
\hline Sphagnum capillifolium & . & . & . & 1 & . & . & 1 & . & 25 \\
\hline Sphagnum rubellum & 1 & 1 & . & . & . & . & . & . & 25 \\
\hline Calypogeia fissa & + & . & . & . & . & . & . & . & 13 \\
\hline \multicolumn{10}{|l|}{ OTHER COMPANIONS } \\
\hline Deschampsia caespitosa & $\mathrm{r}$ & . & 2 & 2 & 1 & 3 & 1 & . & 75 \\
\hline Viola biflora & . & . & . & . & . & 1 & 1 & . & 25 \\
\hline Pellia neesiana & + & . & . & . & . & . & + & . & 25 \\
\hline
\end{tabular}


Tab. 15. Cardamino-Chrysosplenietum alternifolii $(\mathrm{CCh})$. $(\mathrm{D})=$ differential species.

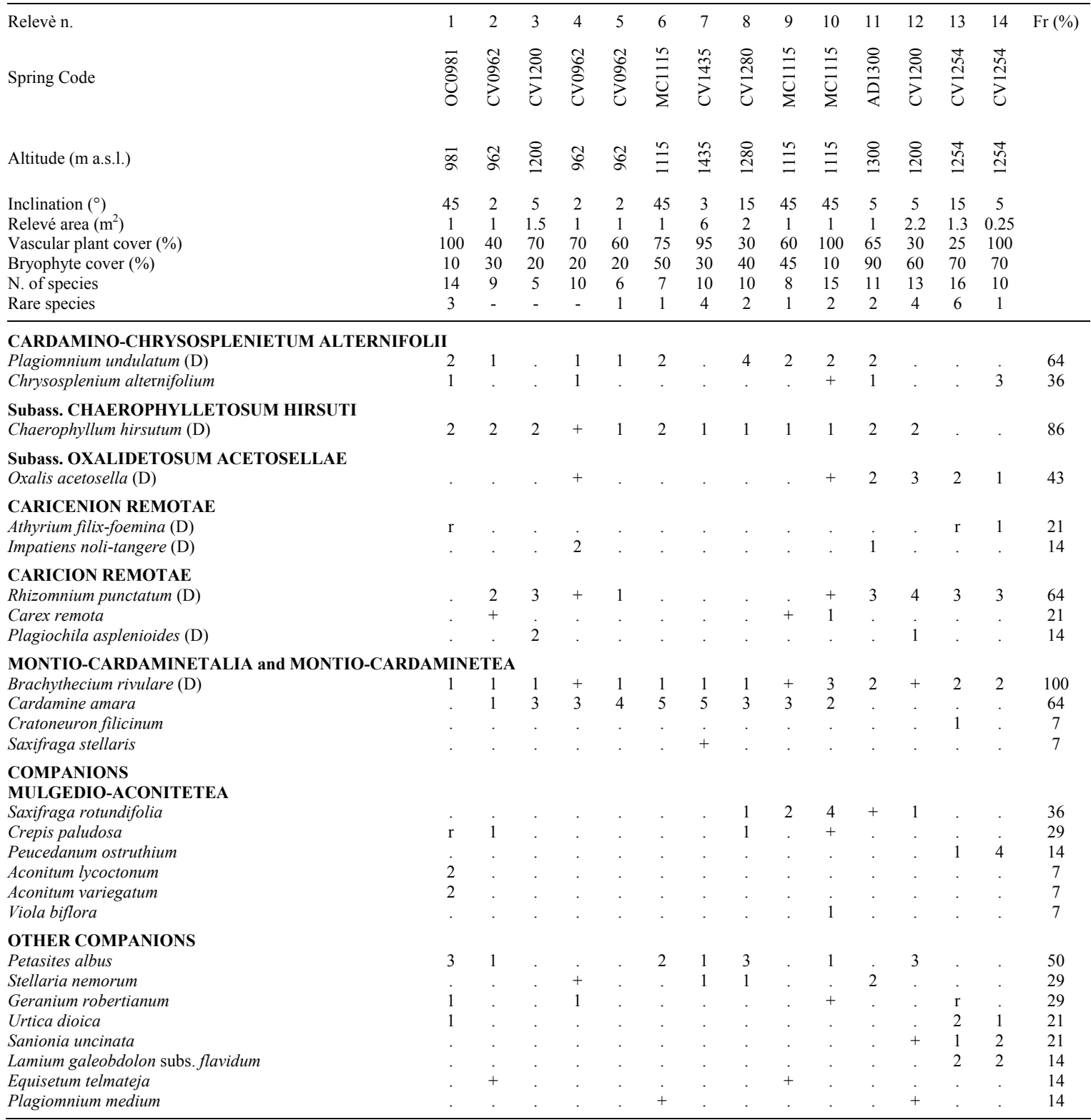


Tab. 16. Eucladietum verticillati (EV). (D) = differential species.

\begin{tabular}{|c|c|c|c|c|}
\hline Relevè n. & 1 & 2 & 3 & $\operatorname{Fr}(\%)$ \\
\hline Spring Code & LD0509 & PG0453 & VF0745 & \\
\hline Altitude (m a.s.1.) & 509 & 453 & 745 & \\
\hline Inclination $\left(^{\circ}\right)$ & 60 & 90 & 55 & \\
\hline Relevé area $\left(\mathrm{m}^{2}\right)$ & 1 & 2.4 & 0.5 & \\
\hline Vascular plant cover $(\%)$ & - & - & 20 & \\
\hline Bryophyte cover $(\%)$ & 50 & 30 & 60 & \\
\hline N. of species & 3 & 5 & 7 & \\
\hline \multicolumn{5}{|l|}{ EUCLADIETUM VERTICILLATI } \\
\hline Eucladium verticillatum & 3 & 3 & 2 & 100 \\
\hline \multicolumn{5}{|c|}{ ADIANTION, ADIANTETALIA and ADIANTETEA } \\
\hline Hymenostylium recurvirostre & . & 4 & 2 & 67 \\
\hline Hymenostylium recurvirostre var. latifolium & . & . & 2 & 33 \\
\hline Pellia endiviifolia (D) & 2 & . & . & 33 \\
\hline \multicolumn{5}{|l|}{ COMPANIONS } \\
\hline Palustriella commutata (D) & 5 & 3 & 3 & 100 \\
\hline \multicolumn{5}{|l|}{ OTHER COMPANIONS } \\
\hline Orthothecium rufescens & . & + & + & 67 \\
\hline Bryum pseudotriquetrum & . & . & 1 & 33 \\
\hline Jungermannia atrovirens & . & 1 & . & 33 \\
\hline Pinguicula alpina & . & . & $\mathrm{r}$ & 33 \\
\hline
\end{tabular}

Tab. 17. Phytocoenon of Pellia endiviifolia (PE).

\begin{tabular}{lc}
\hline Relevè n. & 1 \\
Spring Code & BR1605 \\
Altitude (m a.s.l.) & 1605 \\
Inclination $\left({ }^{\circ}\right)$ & 45 \\
Relevé area $\left(\mathrm{m}^{2}\right)$ & 0.5 \\
Vascular plant cover (\%) & 15 \\
Bryophyte cover (\%) & 70 \\
N. of species & 6 \\
\hline Phytocoenon of PELLIA ENDIVIIFOLIA & \\
Pellia endiviifolia & 4 \\
CRATONEURION COMMUTATI & \\
Palustriella commutata & 1 \\
COMPANIONS & \\
Adenostyles glabra & 1 \\
Eurhynchium praelongum & 1 \\
Jungermannia atrovirens & 1 \\
Sesleria caerulea & 1 \\
\hline
\end{tabular}

Tab. 18. Phytocoenon of Eriophorum angustifolium (EA).

\begin{tabular}{|c|c|c|c|}
\hline Relevè n. & 1 & 2 & $\operatorname{Fr}(\%)$ \\
\hline Spring Code & AD1790 & CV1940 & \\
\hline Altitude (m a.s.1.) & 1790 & 1940 & \\
\hline Inclination $\left({ }^{\circ}\right)$ & 5 & 2 & \\
\hline Relevé area $\left(\mathrm{m}^{2}\right)$ & 3.5 & 1.5 & \\
\hline Vascular plant cover $(\%)$ & 95 & 70 & \\
\hline Bryophyte cover $(\%)$ & 15 & 20 & \\
\hline N. of species & 7 & 2 & \\
\hline $\begin{array}{l}\text { Phytocoenon of ERIOPHORUM ANGUSTIFOLIUM } \\
\text { Eriophorum angustifolium }\end{array}$ & 3 & 4 & 100 \\
\hline $\begin{array}{l}\text { CARICION LASIOCARPAE } \\
\text { SCHEUCHZERIETALIA PALUSTRIS }\end{array}$ & & & \\
\hline $\begin{array}{l}\text { Carex lasiocarpa } \\
\text { Sphagnum contortum }\end{array}$ & $\begin{array}{l}+ \\
+\end{array}$ & . & $\begin{array}{l}50 \\
50\end{array}$ \\
\hline $\begin{array}{l}\text { SCHEUCHZERIO-CARICETEA NIGRAE } \\
\text { Warnstorfia exannulata } \\
\text { Pinguicula vulgaris }\end{array}$ & $\dot{+}$ & $\begin{array}{l}1 \\
.\end{array}$ & $\begin{array}{l}50 \\
50\end{array}$ \\
\hline $\begin{array}{l}\text { COMPANIONS } \\
\text { MONTIO-CARDAMINETEA } \\
\text { Dichodontium palustre }\end{array}$ & 2 & . & 50 \\
\hline $\begin{array}{l}\text { OTHER COMPANIONS } \\
\text { Bryum pseudotriquetrum } \\
\text { Potentilla erecta }\end{array}$ & $\begin{array}{l}+ \\
+\end{array}$ & . & $\begin{array}{l}50 \\
50\end{array}$ \\
\hline
\end{tabular}


Tab. 19. Caricetum nigrae $(\mathrm{CN})$.

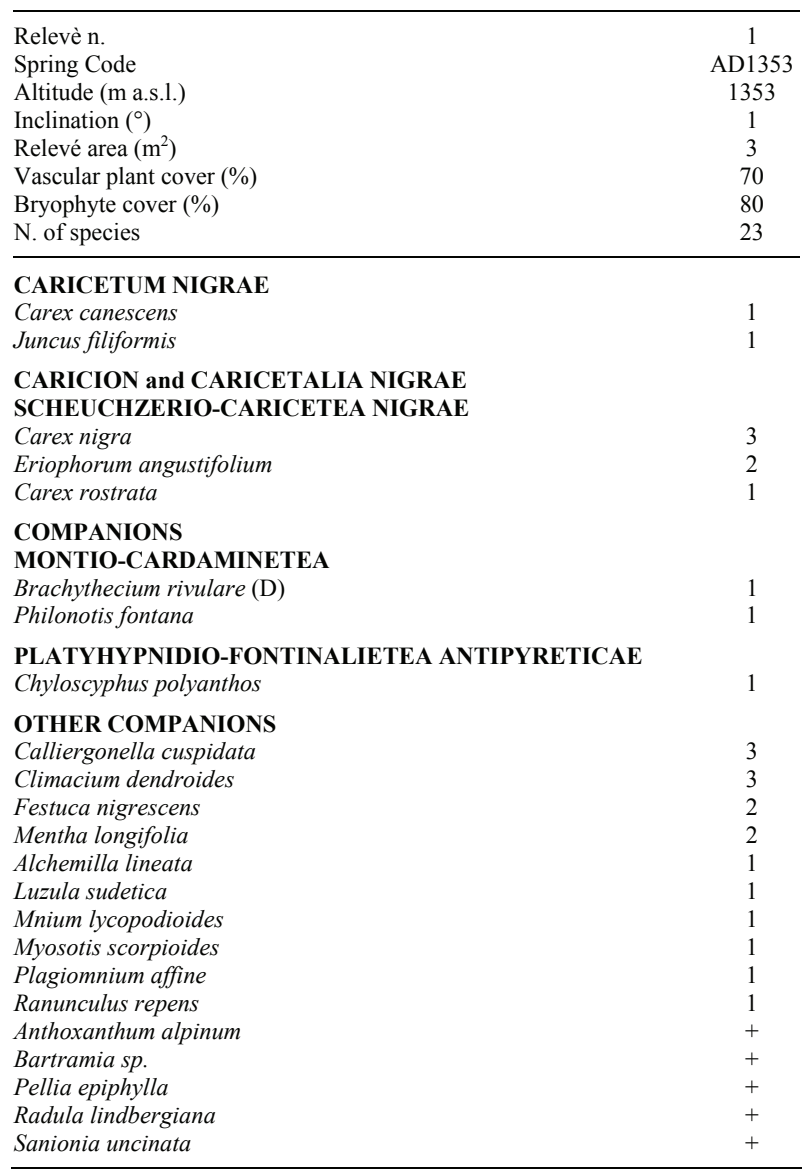

Tab. 20. Phytocoenon of Mentha aquatica (MA). (D) = differential species.

\begin{tabular}{lc}
\hline Relevè n. & 1 \\
Spring Code & BC0503 \\
Altitude (m a.s.l.) & 503 \\
Inclination $\left({ }^{\circ}\right)$ & - \\
Relevé area (m2) & 1.5 \\
Vascular plant cover (\%) & 100 \\
N. of species & 6 \\
\hline Phytocoenon of MENTHA AQUATICA & \\
Mentha aquatica & 4 \\
LEMNA MINOR-FACIES & \\
Lemna minor & 5 \\
CALTHION & + \\
Lysimachia vulgaris & $\mathrm{r}$ \\
Epilobium parviflorum & \\
MOLINIETALIA and MOLINIO-ARRHENATHERETEA & 1 \\
Galium palustre (D) & + \\
Equisetum palustre & \\
\hline
\end{tabular}


Tab. 21. Phytocoenon of Eupatorium cannabinum (EC). (D) = differential species.

\begin{tabular}{lc}
\hline Relevè $\mathrm{n}$. & 1 \\
Spring Code & BR0790 \\
Altitude (m a.s.1.) & 790 \\
Inclination $\left({ }^{\circ}\right)$ & 60 \\
Relevé area $\left(\mathrm{m}^{2}\right)$ & 3 \\
Vascular plant cover (\%) & 95 \\
Bryophyte cover (\%) & 60 \\
N. of species & 7 \\
\hline Phytocoenon of EUPATORIUM CANNABINUM & \\
Eupatorium cannabinum & 4 \\
CONVOLVULETALIA SEPIUM & \\
Mentha longifolia (D) & 3 \\
GALIO-URTICETEA & \\
Geranium robertianum & 1 \\
Urtica dioica & $\mathrm{r}$ \\
COMPANIONS & \\
MONTIO-CARDAMINETEA & \\
Palustriella decipiens & \\
OTHER COMPANIONS & \\
Arrhenatherum elatius & \\
Plagiomnium rostratum & \\
\hline
\end{tabular}

Tab. 22. Chaerophyllo-Petasitetum officinalis (CP). (D) = differential species.

\begin{tabular}{lc}
\hline Relevè n. & 1 \\
Spring Code & CS1350 \\
Altitude (m a.s.l.) & 1350 \\
Inclination $\left({ }^{\circ}\right)$ & 35 \\
Relevé area $\left(\mathrm{m}^{2}\right)$ & 2.3 \\
Vascular plant cover (\%) & 100 \\
Bryophyte cover (\%) & 10 \\
N. of species & 8 \\
\hline CHAEROPHYLLO-PETASITETUM OFFICINALIS & \\
Mentha longifolia (D) & 4 \\
Petasites hybridus & 3 \\
PETASITION OFFICINALIS & \\
Cardamine amara (D) & 3 \\
Chaerophyllum hirsutum (D) & 1 \\
LAMIO ALBI-CHENOPODIETALIA BONI-HENRICI & \\
GALIO-URTICETEA & \\
Urtica dioica & 1 \\
COMPANIONS & \\
MONTIO-CARDAMINETEA & \\
Cratoneuron filicinum & \\
Brachythecium rivulare (D) & \\
OTHER COMPANIONS & \\
Equisetum arvense & \\
\hline
\end{tabular}


Tab. 23. Phytocoenon of Mentha longifolia (ML). (D) $=$ differential species.

\begin{tabular}{|c|c|c|c|}
\hline Relevè n. & 1 & 2 & $\operatorname{Fr}(\%)$ \\
\hline Spring Code & MB1440 & MB1440 & \\
\hline Altitude (m a.s.1.) & 1440 & 1440 & \\
\hline Inclination $\left({ }^{\circ}\right)$ & 20 & 10 & \\
\hline Relevé area $\left(\mathrm{m}^{2}\right)$ & 1 & 1 & \\
\hline Vascular plant cover $(\%)$ & 90 & 100 & \\
\hline Bryophyte cover $(\%)$ & 5 & 5 & \\
\hline N. of species & 11 & 10 & \\
\hline \multicolumn{4}{|l|}{ Phytocoenon of MENTHA LONGIFOLIA } \\
\hline Mentha longifolia & 3 & 3 & 100 \\
\hline \multicolumn{4}{|c|}{ RUMICION and RUMICETALIA ALPINI } \\
\hline Chaerophyllum hirsutum & 2 & 2 & 100 \\
\hline Saxifraga rotundifolia & 2 & 1 & 100 \\
\hline Adenostyles alliariae & 1 & 2 & 100 \\
\hline Alchemilla hirtipes (D) & $\mathrm{r}$ & 2 & 100 \\
\hline Viola biflora & + & 1 & 100 \\
\hline Geum rivale & . & 2 & 50 \\
\hline \multicolumn{4}{|l|}{$\begin{array}{l}\text { COMPANIONS } \\
\text { CRATONEURION COMMUTATI }\end{array}$} \\
\hline Palustriella decipiens & 1 & . & 50 \\
\hline Cratoneuron filicinum & + & . & 50 \\
\hline \multicolumn{3}{|l|}{ MONTIO-CARDAMINETEA } & 50 \\
\hline \multicolumn{4}{|l|}{ OTHER COMPANIONS } \\
\hline Myosotis sylvatica & $\mathrm{r}$ & 2 & 100 \\
\hline Cerastium arvense subsp. suffruticosum & 1 & 1 & 100 \\
\hline Poa alpina & . & + & 50 \\
\hline
\end{tabular}

\subsection{Ecological analysis}

\subsubsection{Variation range of environmental parameters}

The environmental parameters show different ranges among the vegetation types (Tab. 24). The altitudinal range is very broad, between 170 and $2739 \mathrm{~m}$ a.s.l. Most crenic vegetation types are, however, concentrated within the montane vegetation belt, from 800 to $1600 \mathrm{~m}$. Shading is also very variable. Most vegetation types belong to classes 3 and 4, corresponding to more than $50 \%$ shading. Only Solenostomo-Hygrohypnetum smithii and Blindio-Scapanietum undulatae are restricted to the very sunny sites of class 1 (shading $0-25 \%$ ).

Conductivity and alkalinity also show similar distribution patterns, with the vegetation types clearly separated into two distinct groups. The first, including the majority of types, has between 200 and $400 \mu \mathrm{S} \mathrm{cm}^{-1}$ conductivity and 100 and $200 \mathrm{mg} \mathrm{L}^{-1} \mathrm{CaCO}_{3}$ alkalinity. It comprises all types around springs flowing through carbonate bedrock. In the second group, conductivity

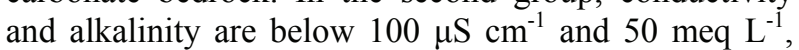
respectively. All these vegetation types occur on siliceous bedrocks. The variance of conductivity and alkalinity within the same vegetation type is generally low, apart from the Cratoneuron filicinum phytocoenon, in which both parameters are enhanced in two stands by excessive nitrate. $\mathrm{pH}$ shows a similar distribution pattern, with the vegetation types on carbonate substrata having $\mathrm{pH}$ values around 8.0. The vegetation types on siliceous massifs show a clear trend toward higher variation between stands, especially the Cardamino-
Chrysosplenietum alternifolii and the Montio-Bryetum schleicheri, where $\mathrm{pH}$ ranges from 6.5 and 7.5 , and the Blindio-Scapanietum undulatae, whose stands have $\mathrm{pH}$ values between 5.9 and 7.0. Sulphate behaves very peculiarly, because six vegetation types have very high internal variation, whereas all the other ones have low sulphate values (below $10 \mathrm{mg} \mathrm{L}^{-1}$ ). However the high sulphate values of some vegetation are explained by one or more relevés from sulphurous springs.

Nutrient availability for the different vegetation types is shown in the nitrate and phosphate columns. Nitrate concentration is below the mesotrophic-eutrophic boundary for all the vegetation types, with the exception of the Mentha longifolia phytocoenon. The majority of vegetation types are irrigated by oligotrophic crenic waters, while a few have continuous irrigation with mesotrophic waters. The broad variation within the Cratoneuron filicinum phytocoenon is determined by the relevé from a spring close to a farm (see above). The phosphate column shows that all vegetation types have less than $25 \mu \mathrm{g} \mathrm{L}^{-1}$ available-P, corresponding to the oligotrophic-mesotrophic boundary. Most vegetation types occur in springs with water with less than $5 \mu \mathrm{g} \mathrm{L}^{-1}$ phosphate. The highest values are recorded for some tall herb communities (including Chaerophyllo-Cardaminetum asarifoliae and the Cardamino-Chrysosplenietum alternifolii subass. oxalidetosum acetosellae).

Flow velocity in crenic habitats is a synthetic variable, variously influenced by discharge and slope, and therefore both discharge and flow velocity should be 
Tab. 24. Median, lower and upper quartile (25th -75 th ) of the main environmental variables in the phytosociological units.

\begin{tabular}{|c|c|c|c|c|c|c|c|c|c|c|c|c|c|c|c|c|}
\hline \multirow[t]{2}{*}{ phytosociological unit } & \multirow[t]{2}{*}{$\mathrm{N}$} & \multicolumn{3}{|c|}{ Altitude (m a.s.1.) } & \multicolumn{3}{|c|}{ Shading class } & \multicolumn{3}{|c|}{ El. cond. $\left(\mu \mathrm{S} \mathrm{cm}^{-1}\right)$} & \multicolumn{3}{|c|}{ Alkal $\left(\mathrm{mg} \mathrm{L}^{-1} \mathrm{CaCO}_{3}\right)$} & \multicolumn{3}{|c|}{$\mathrm{pH}$} \\
\hline & & $\overline{\text { median }}$ & 25 th & 75th & median & 25 th & 75 th & median & 25 th & 75 th & median & 25 th & 75 th & median & 25 th & 75 th \\
\hline 1. Scapanietum undulatae & 4 & 1387 & 1353 & 1428 & 2.5 & 2.0 & 3.5 & 33 & 31 & 34 & 11.5 & 9.5 & 12.0 & 6.77 & 6.67 & 6.84 \\
\hline 2. Oxyrrhynchietum rusciformis & 18 & 633 & 470 & 992 & 4.0 & 3.0 & 4.0 & 267 & 232 & 313 & 143.5 & 103.0 & 174.0 & 7.92 & 7.67 & 8.00 \\
\hline 3. Cinclidotetum aquatici & 1 & 686 & 686 & 686 & 3.0 & 3.0 & 3.0 & 180 & 180 & 180 & 104.0 & 104.0 & 104.0 & 7.88 & 7.88 & 7.88 \\
\hline 4. Brachythecio rivularis-Hygrohypnetum luridi & 7 & 1300 & 656 & 1315 & 3.0 & 3.0 & 4.0 & 225 & 207 & 297 & 130.0 & 118.0 & 132.0 & 7.96 & 7.91 & 8.04 \\
\hline 5. Phytocoenon of Hygroamblystegium tenax & 4 & 360 & 210 & 470 & 3.5 & 3.0 & 4.0 & 269 & 252 & 294 & 112.0 & 103.0 & 140.5 & 7.92 & 7.83 & 8.00 \\
\hline 6. Cratoneuretum commutati & 21 & 1000 & 790 & 1474 & 4.0 & 3.0 & 4.0 & 345 & 241 & 392 & 149.0 & 131.0 & 220.0 & 7.97 & 7.91 & 8.09 \\
\hline 7. Cratoneuretum falcati & 15 & 1502 & 1400 & 1765 & 3.0 & 2.0 & 4.0 & 212 & 179 & 221 & 120.0 & 98.0 & 132.0 & 8.04 & 7.88 & 8.14 \\
\hline 8. Phytocoenon of Cratoneuron filicinum & 7 & 590 & 565 & 1350 & 2.0 & 2.0 & 4.0 & 301 & 204 & 796 & 170.0 & 149.0 & 437.0 & 7.94 & 7.47 & 8.04 \\
\hline 9. Phytocoenon of Palustriella decipiens & 1 & 1670 & 1670 & 1670 & 4.0 & 4.0 & 4.0 & 223 & 223 & 223 & 123.0 & 123.0 & 123.0 & 7.87 & 7.87 & 7.87 \\
\hline 10. Chaerophyllo-Cardaminetum asarifoliae & 1 & 1235 & 1235 & 1235 & 4.0 & 4.0 & 4.0 & 198 & 198 & 198 & 109.0 & 109.0 & 109.0 & 8.03 & 8.03 & 8.03 \\
\hline 11. Phytocoenon of Petasites albus & 4 & 979 & 782 & 1290 & 3.5 & 2.0 & 4.0 & 293 & 227 & 358 & 165.4 & 112.4 & 217.5 & 7.96 & 7.69 & 8.17 \\
\hline 12. Montio-Bryetum schleicheri & 18 & 1911 & 1623 & 1990 & 1.5 & 1.0 & 2.0 & 33 & 28 & 60 & 11.7 & 7.9 & 17.0 & 6.86 & 6.60 & 7.03 \\
\hline 13. Solenostomo-Hygrohypnetum smithii & 4 & 2314 & 2234 & 2527 & 1.0 & 1.0 & 1.0 & 19 & 18 & 23 & 4.4 & 3.8 & 7.5 & 6.54 & 6.29 & 6.80 \\
\hline 14. Blindio-Scapanietum undulatae & 8 & 1899 & 1748 & 2102 & 1.0 & 1.0 & 1.0 & 16 & 12 & 22 & 3.6 & 3.2 & 6.8 & 6.17 & 5.93 & 6.40 \\
\hline 15. Cardamino-Chrysosplenietum alternifolii & 14 & 1158 & 981 & 1254 & 4.0 & 4.0 & 4.0 & 72 & 44 & 120 & 28.5 & 19.0 & 56.7 & 7.17 & 6.85 & 7.54 \\
\hline 16. Eucladietum verticillati & 3 & 509 & 453 & 745 & 4.0 & 1.0 & 4.0 & 333 & 240 & 340 & 177.0 & 138.0 & 205.0 & 7.87 & 7.86 & 8.19 \\
\hline 17. Phytocoenon of Pellia endiviifolia & 1 & 1605 & 1605 & 1605 & 2.0 & 2.0 & 2.0 & 241 & 241 & 241 & 147.0 & 147.0 & 147.0 & 8.32 & 8.32 & 8.32 \\
\hline 18. Phytocoenon of Eriophorum angustifolium & 2 & 1865 & 1790 & 1940 & 1.5 & 1.0 & 2.0 & 34 & 28 & 39 & 12.8 & 10.5 & 15.0 & 6.90 & 6.85 & 6.95 \\
\hline 19. Caricetum nigrae & 1 & 1353 & 1353 & 1353 & 2.0 & 2.0 & 2.0 & 33 & 33 & 33 & 12.0 & 12.0 & 12.0 & 6.84 & 6.84 & 6.84 \\
\hline 20. Phytocoenon of Mentha aquatica & 1 & 503 & 503 & 503 & 4.0 & 4.0 & 4.0 & 241 & 241 & 241 & 131.0 & 131.0 & 131.0 & 7.98 & 7.98 & 7.98 \\
\hline 21. Phytocoenon of Eupatorium cannabinum & 1 & 790 & 790 & 790 & 4.0 & 4.0 & 4.0 & 368 & 368 & 368 & 220.0 & 220.0 & 220.0 & 7.91 & 7.91 & 7.91 \\
\hline yllo-Petasitetum & 1 & 1350 & 1350 & 1350 & 2.0 & 2.0 & 2.0 & 301 & 301 & 301 & 170.0 & 170.0 & 170.0 & 8.20 & 8.20 & 8.20 \\
\hline 23. Phytocoenon of Mentha longifolia & 2 & 1440 & 1440 & 1440 & 2.0 & 2.0 & 2.0 & 261 & 261 & 261 & 143.0 & 143.0 & 143.0 & 8.07 & 8.07 & 8.07 \\
\hline \multirow[t]{2}{*}{ phytosociological unit } & \multirow[t]{2}{*}{$\mathrm{N}$} & \multicolumn{3}{|c|}{$\mathrm{SO}_{4}\left(\mathrm{mg} \mathrm{L}^{-1}\right)$} & \multicolumn{3}{|c|}{$\mathrm{N}-\mathrm{NO}_{3}\left(\mu \mathrm{g} \mathrm{L}^{-1}\right)$} & \multicolumn{3}{|c|}{$\mathrm{P}-\mathrm{PO}_{4}\left(\mu \mathrm{g} \mathrm{L}^{-1}\right)$} & \multicolumn{3}{|c|}{ Discharge $\left(\mathrm{L} \mathrm{s}^{-1}\right)$} & \multicolumn{3}{|c|}{ Flow vel. $\left(\mathrm{cm} \mathrm{s}^{-1}\right)$} \\
\hline & & $\overline{\text { median }}$ & 25 th & 75 th & median & 25 th & 75 th & median & 25 th & 75 th & median & 25 th & 75 th & median & 25 th & 75 th \\
\hline 1. Scap & 4 & 2.1 & 1.8 & 3.0 & 864 & 483 & 1012 & 1.3 & 1.2 & 3.8 & 12.5 & 3.0 & 20.0 & 34 & 10 & 53 \\
\hline 2. Oxyrrhynchietum rusciformis & 18 & 2.8 & 2.1 & 5.6 & 709 & 361 & 1197 & 5.2 & 1.6 & 7.0 & 4.5 & 3.0 & 7.0 & 9 & 5 & 13 \\
\hline 3. Cinclidotetum aquatici & 1 & 1.9 & 1.9 & 1.9 & 333 & 333 & 333 & 4.4 & 4.4 & 4.4 & 120.0 & 120.0 & 120.0 & 1 & 1 & 1 \\
\hline 4. Brachythecio rivularis-Hygrohypnetum luridi & 7 & 5.6 & 3.0 & 35.5 & 659 & 309 & 738 & 3.0 & 1.3 & 3.5 & 2.3 & 1.0 & 7.0 & 25 & 5 & 50 \\
\hline 5. Phytocoenon of Hygroamblystegium tenax & 4 & 4.1 & 1.3 & 15.4 & 665 & 427 & 1498 & 3.8 & 2.6 & 10.2 & 16.5 & 2.0 & 30.0 & 0 & 0 & 10 \\
\hline 6. Cratoneuretum commutati & 21 & 7.4 & 4.7 & 16.6 & 597 & 318 & 768 & 1.5 & 1.2 & 2.5 & 0.5 & 0.3 & 1.5 & 15 & 10 & 20 \\
\hline 7. Cratoneuretum falcati & 15 & 3.1 & 1.3 & 7.1 & 543 & 247 & 746 & 2.3 & 1.1 & 2.5 & 1.0 & 0.3 & 3.0 & 20 & 10 & 40 \\
\hline 8. Phytocoenon of Cratoneuron filicinum & 7 & 6.8 & 5.4 & 33.0 & 893 & 803 & 6885 & 6.9 & 5.5 & 13.1 & 1.0 & 0.8 & 1.0 & 10 & 4 & 20 \\
\hline 9. Phytocoenon of Palustriella decipiens & 1 & 6.6 & 6.6 & 6.6 & 605 & 605 & 605 & 0.9 & 0.9 & 0.9 & 0.5 & 0.5 & 0.5 & 10 & 10 & 10 \\
\hline 10. Chaerophyllo-Cardaminetum asarifoliae & 1 & 3.8 & 3.8 & 3.8 & 973 & 973 & 973 & 9.8 & 9.8 & 9.8 & 0.9 & 0.9 & 0.9 & 20 & 20 & 20 \\
\hline 11. Phytocoenon of Petasites albus & 4 & 3.0 & 2.1 & 6.0 & 458 & 212 & 694 & 2.9 & 1.9 & 6.9 & 0.5 & 0.3 & 3.8 & 18 & 3 & 30 \\
\hline 12. Montio-Bryetum schleicheri & 18 & 3.2 & 1.9 & 6.4 & 312 & 231 & 464 & 2.8 & 2.0 & 6.0 & 1.0 & 0.2 & 2.5 & 18 & 7 & 30 \\
\hline 13. Solenostomo-Hygrohypnetum smithii & 4 & 1.0 & 0.9 & 1.2 & 843 & 437 & 1137 & 1.2 & 1.2 & 2.0 & 0.6 & 0.5 & 7.9 & 28 & 8 & 40 \\
\hline 14. Blindio-Scapanietum undulatae & 8 & 1.5 & 1.1 & 3.3 & 254 & 197 & 301 & 1.0 & 1.0 & 1.2 & 2.9 & 1.5 & 3.3 & 40 & 8 & 45 \\
\hline 15. Cardamino-Chrysosplenietum alternifolii & 14 & 3.7 & 2.5 & 5.2 & 361 & 108 & 717 & 6.1 & 2.3 & 7.0 & 0.3 & 0.2 & 3.0 & 15 & 10 & 25 \\
\hline 16. Eucladietum verticillati & 3 & 4.3 & 2.3 & 18.9 & 915 & 654 & 969 & 1.0 & 0.0 & 1.0 & 0.3 & 0.2 & 2.0 & 25 & 20 & 30 \\
\hline 17. Phytocoenon of Pellia endiviifolia & 1 & 1.9 & 1.9 & 1.9 & 238 & 238 & 238 & 1.2 & 1.2 & 1.2 & 0.3 & 0.3 & 0.3 & 40 & 40 & 40 \\
\hline 18. Phytocoenon of Eriophorum angustifolium & 2 & 1.6 & 1.2 & 2.0 & 716 & 297 & 1135 & 2.8 & 2.4 & 3.1 & 0.5 & 0.2 & 0.7 & 23 & 20 & 25 \\
\hline 19. Caricetum nigrae & 1 & 1.8 & 1.8 & 1.8 & 1012 & 1012 & 1012 & 1.3 & 1.3 & 1.3 & 20.0 & 20.0 & 20.0 & 53 & 53 & 53 \\
\hline 20. Phytocoenon of Mentha aquatica & 1 & 10.2 & 10.2 & 10.2 & 333 & 333 & 333 & 2.0 & 2.0 & 2.0 & 3.0 & 3.0 & 3.0 & 5 & 5 & 5 \\
\hline 21. Phytocoenon of Eupatorium cannabinum & 1 & 2.7 & 2.7 & 2.7 & 318 & 318 & 318 & 5.5 & 5.5 & 5.5 & 1.0 & 1.0 & 1.0 & 15 & 15 & 15 \\
\hline 22. Chaerophyllo-Petasitetum officinalis & 1 & 6.8 & 6.8 & 6.8 & 893 & 893 & 893 & 13.1 & 13.1 & 13.1 & 1.0 & 1.0 & 1.0 & 22 & 22 & 22 \\
\hline 23. Phytocoenon of Mentha longifolia & 2 & 3.8 & 3.8 & 3.8 & 1823 & 1823 & 1823 & 7.5 & 7.5 & 7.5 & 0.2 & 0.2 & 0.2 & 10 & 10 & 10 \\
\hline
\end{tabular}

considered. Most vegetation types occur at springs with less than $5 \mathrm{~L} \mathrm{~s}^{-1}$ discharge. The bryophyte-dominated vegetation types belonging to the class PlatyhypnidioFontinalietea antipyreticae were encountered at springs with the highest discharge and variation. Among them, the Cinclidotetum aquatici was sampled inside a spring with the highest values for both discharge and flow velocity, where the dominant moss is totally submerged. On the other hand, most bryophyte and vascular species in the Caricetum nigrae are non-submerged around the helocrene, where flow velocity is lower despite the constant high discharge.

\subsubsection{Discriminant Analysis}

Discriminant analysis of the environmental variables among the vegetation types showed that the first three canonical variates were highly significant, and accounted for more than $88 \%$ cumulative proportion of explained variance (Tab. 25). The factor structure coefficients for the first three canonical variates (Tab. 25) indicated that the environmental descriptors that best accounted for the among-group variation were $\mathrm{pH}$ and alkalinity, and altitude (first variate); altitude and discharge (second variate); alkalinity and nitrate, and shading (third variate). Figure 2 shows the position of the centroids of the 20 vegetation types in relation to the first two canonical axes. According to figure 2, the vegetation types were mainly distributed along three gradients. The first gradient, corresponding to the first axis, reflects differences in $\mathrm{pH}$ and alkalinity; the second axis separated the vegetation types occurring at high altitude (positive values) and those with a high dis- 
Tab. 25. Structure coefficients for the first three significant canonical variates. Values in bold are the most significant. See text for details.

\begin{tabular}{lccc}
\hline & Canonical variate I & Canonical variate II & Canonical variate II \\
\hline Wilk's $\Lambda$ & 0.037 & 0.129 & 0.271 \\
$\chi^{2}$ & 383.2 & 237.8 & 151.4 \\
$\mathrm{df}$ & 144 & 120 & 98 \\
$\mathrm{P}$ & $>0.001$ & $>0.001$ & $>0.001$ \\
Discriminant variable & & & \\
altitude & $\mathbf{0 . 4 7 5}$ & $\mathbf{0 . 5 9 5}$ & -0.266 \\
shading & 0.274 & -0.126 & $\mathbf{0 . 5 4 8}$ \\
conductivity & 0.190 & 0.112 & -0.129 \\
alcalinity & $\mathbf{0 . 5 4 0}$ & 0.107 & $\mathbf{- 0 . 5 2 6}$ \\
pH & $\mathbf{0 . 6 4 3}$ & 0.276 & 0.069 \\
sulphate & 0.030 & 0.104 & 0.011 \\
nitrate & 0.110 & -0.125 & $\mathbf{- 0 . 5 0 6}$ \\
phosphate & 0.039 & -0.246 & -0.208 \\
discharge & 0.060 & $\mathbf{- 0 . 3 4 7}$ & 0.122 \\
flow velocity & -0.066 & 0.168 & 0.025 \\
Eigenvalue & 9.264 & 2.500 & 1.107 \\
Cum. Prop. & 0.639 & 0.812 & 0.888 \\
\hline
\end{tabular}

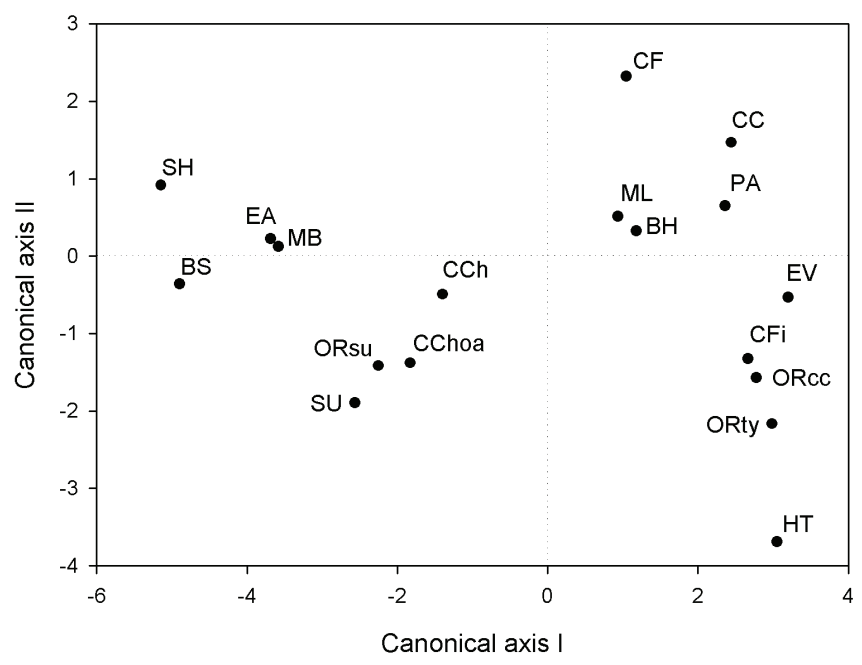

Fig. 2. Centroids in the canonical discriminant space of the vegetation types occurring in the investigated spring habitats (only the vegetation types with more than two cases were considered, see text for acronyms and more details).

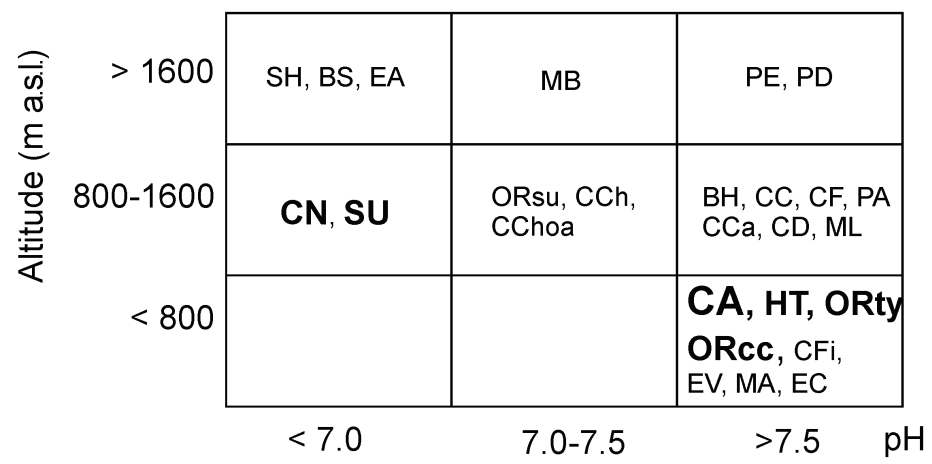

Fig. 3. Schematic diagram showing the arrangement of vegetation types along the main environmental gradients: altitude, $\mathrm{pH}$ and discharge. Boldface acronyms correspond to vegetation types occurring at springs with higher discharge. Acronyms are those reported in the text.

charge (negative values). A schematic diagram (Fig. 3) shows how the syntaxa were arranged across the three main gradients, altitude, $\mathrm{pH}$ and discharge. About 55\% of vegetation types have alkaline waters with $\mathrm{pH}$ above 7.5, distributed below $1600 \mathrm{~m}$ a.s.1.; whereas all the vegetation types with neutral or acid waters, $\mathrm{pH}$ less than 7.0, occur above $800 \mathrm{~m}$ a.s.l. These patterns can be explained by the different altitudinal distribution of carbonate and siliceous substrata in the study area. The vegetation types within springs with high discharge are 
printed in bold. The majority of them occur at low altitudes and almost all belong to the class Platyhypnidio-Fontinalietea antipyreticae.

\section{DISCUSSION AND CONCLUSIONS}

The syntaxonomical arrangement of crenic vegetation in Trentino is synthesized in table 26. It includes 23 plant communities, 14 typified as associations (two of them including also two or more subassociations), and 9 arranged as phytocoena. The high number of phytocoena is reasonable for plant communities often characterized by extreme paucity of their floristic assemblage, where the dominant species may have only a weak diagnostic value.
The classes Platyhypnidio-Fontinalietea antipyreticae and Montio-Cardaminetea subsume most of what is regarded as "crenic vegetation". They include about two thirds of the vegetation types identified here, of which almost two thirds belong to the class Montio-Cardaminetea, comprising non-submerged plant communities containing variable mixtures of bryophytes and vascular plants. The Cratoneurion commutati alliance, represented by 6 different vegetation types, forms the core of the class. The two commonest and most widespread plant communities of the alliance are the Cratoneuretum commutati and the Cratoneuretum falcati, with well-defined peaks of altitudinal occurrence, but, in spite of this, reciprocally connected along the altitudinal gradient by subcommunities.

Tab. 26. Syntaxonomical scheme.

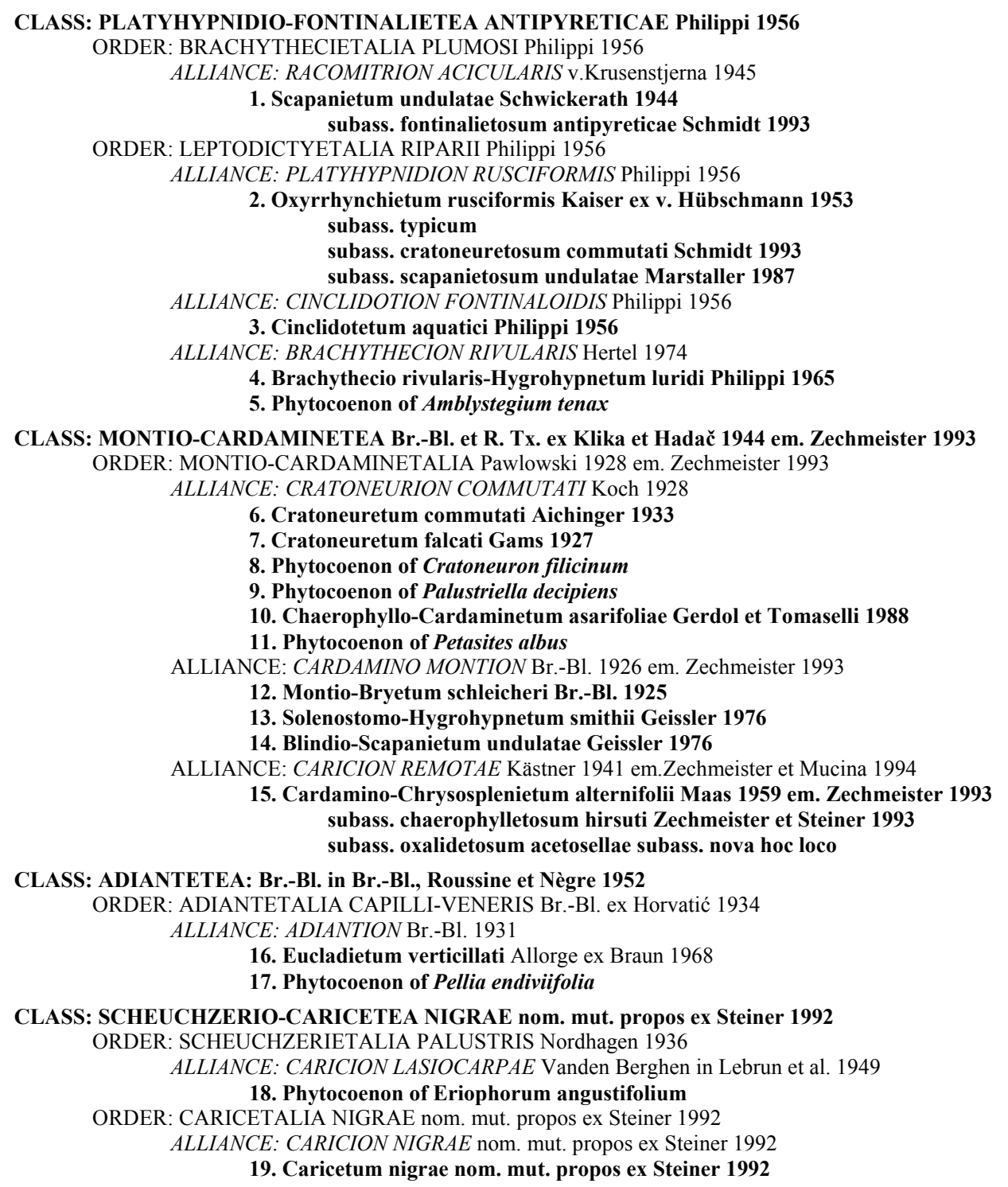


Tab. 26. Continuation.

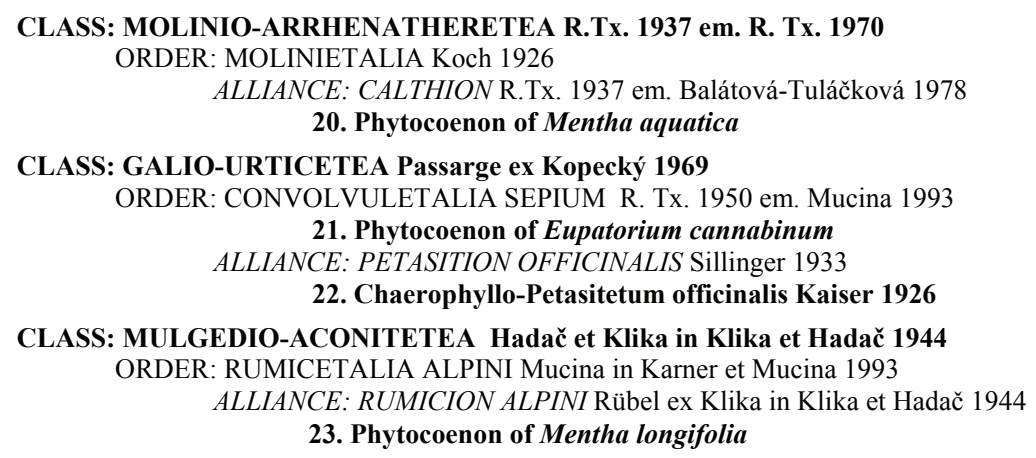

The other communities within the Cratoneurion commutati are scattered throughout the study area. Moreover, they are not as clearly characterized from a floristical viewpoint, except the Chaerophyllo-Cardaminetum asarifoliae. This association has been briefly mentioned in the literature, reported only from the northern Apennines, but it seems likely that its full range roughly matches that of the dominant species, including the Western Alps, with scattered localities in the Eastern Alps, as far as the Autonomous Province of Trento.

The Cardamino-Montion alliance appears with only 3 associations, restricted to the upper montane to the alpine vegetation belt. They represent little under half the associations of this alliance reported from the Alps. This could be explained by the low occurrence of highaltitude helocrene-type springs on the siliceous massifs, further supported by the complete absence of crenic communities belonging to the alliance Dermatocarpion .

The Caricion remotae alliance is also under-represented in the study area, where it occurs with only one association. Caricion remotae communities are strictly linked to montane shaded springs, with acidic to circumneutral waters. Their occurrence in Trentino is, therefore, restricted to the porphyritic rocks of the Lagorai range, and inhibited elsewhere by limestone rocks. On the other hand, the carbonate lithology prevailing within the collinar and montane vegetation belts enhances the occurrence of basiphilous communities belonging to the class Platyhypnidio-Fontinalietea antipyreticae. The most widespread and floristically diversified association is the Oxyrrhynchietum rusciformis.

From its core, represented by the classes Platyhypnidio-Fontinalietea antipyreticae and Montio-Cardaminetea, crenic vegetation shows a number of floristic trends towards other physically and ecologically "peripheral" communities. Among them, those belonging to the Adiantion and to the Scheuchzerio-Caricetea nigrae have the closest affinities with the "true" crenic vegetation. The more or less nitrophilous, tall herb communities have their coenological foci outside springs. They occur more frequently within small springs, where boundary delimitation with the surrounding habitats is problematic.

The ecological analysis revealed that phytocoenological diversity within the crenic vegetation sampled in Trentino can be mainly explained by abiotic variables, such as alkalinity and $\mathrm{pH}$, reflecting the nature of the substrata from which springs emerge. Temperature, mirrored by altitude, also has a strong influence on the floristic composition of crenic communities. Discharge mainly explains the floristic differences between the submerged bryophyte communities and the others. Floristic differences among the communities of the class Platyhypnidio-Fontinalietea antipyreticae are also conditioned by discharge. In general, as reported in Spitale et al. (2009), discharge affects crenic complexity, area and species richness. The largest springs with higher discharge have maximal floristic richness and phytocoenological diversity.

When discharge is reduced by more or less intensive water-collection, as frequently happens in lower areas, crenic area and species richness are, in turn, reduced. In these cases, vegetation looses its typical features and becomes a mosaic of fragmentary small stands, in which the original crenic species are replaced by "invader" species from the surrounding habitats.

\section{ACKNOWLEDGMENTS}

This work was financed by the University and Scientific Research Department of the Autonomous Province of Trento (CRENODAT Project: Biodiversity assessment and integrity evaluation of springs of Trentino - Italian Alps - and long-term ecological research, 2004-2008).

\section{REFERENCES}

Aeschimann, D., K. Lauber, D.M. Moser \& J.P. Theurillat. 2004. Flora alpina. 3. Zanichelli, Bologna: 2670 pp.

APHA. 2000. Standard Methods for the Examination of Water and Wastewater. 20th ed., APHA, AWWA \& WEF, American Public Health Association,Washington D.C.

Birse, E.L. 1980. Plant communities of Scotland: a preliminary phytocoenonia. Macaulay Institute for Soil Research, Aberdeen. 
Boiti, I., C. Lasen \& T. Saffaro Boiti. 1989. La vegetazione della Val Venegia. Ed. Manfrini, Calliano (TN): 164 pp.

Braun-Blanquet, G. \& J. Braun-Blanquet. 1931. Recherches phytogeographiques sur le Massif du Grossglockner (Hohe Tauern). S.I.G.M.A. Comm., Montpellier, 13: 1-65.

Braun-Blanquet, J. 1954. La végétation alpine et nivale des Alpes françaises. Etude botanique de l'étage alpin. S.I.G.M.A. Comm., Montpellier, 125: 1-72.

Braun-Blanquet, J. 1964. Pflanzensoziologie. 3. Aufl. Springer, Wien: $865 \mathrm{pp}$.

Cantonati, M., R. Gerecke \& E. Bertuzzi 2006. Springs of the Alps, sensitive ecosystems to environmental change: from biodiversity assessments to long-term studies. Hydrobiologia, 562: 59-96.

Deil, U. 1996. Zur Kenntnis der Klasse Adiantetea des Mittelmeerraumes und angrenzender Gebiete mit allgemeinen Überlegungen zur ökologischen Skalierung ihrer Standorte und zur Sättigung von Pflanzengesellschaften. Phytocoenologia, 26: 481-536.

Dierßen, K. 1982. Die wichtigsten Pflanzengesellschaften der Moore NW Europas. Conservatoires et Jardin Botanique, Genève: $382 \mathrm{pp}$.

Dierßen, K. 1996. Vegetation Nordeuropas. Ulmer, Stuttgart: $838 \mathrm{pp}$

Dierßen, K. 2001. Distribution, ecological amplitude and phytosociological characterization of European bryophytes. Bryophytorum Bibliotheca Band 56. Cramer, Berlin: $289 \mathrm{pp}$.

Dirnböck, T., S. Dullinger, M. Gottfried \& G. Grabherr. 1999. Die Vegetation des Hochschwab (Steiermark). Alpine und Subalpine stufe. Mitt. naturwiss. Ver. Steiermark, 129: 111-251.

Geissler, P. 1976. Zur Vegetation alpiner Fließgewässer. Beitr. Kryptogamenflora Schweiz, 14: 1-52.

Gerdol, R. 1994. The vegetation of wetlands in the southern Carnian Alps (Italy). Gortania - Atti Mus. Friul. Storia Nat., 15: 67-107.

Gerdol, R. \& M. Tomaselli. 1988. Phytosociology and ecology of stream vegetation in the summit region of the northern Apennines. Boll. Mus. St. Nat. Lunigiana, 6-7 (19861987): 89-93.

Gerdol, R. \& M. Tomaselli. 1997. Vegetation of wetlands in the Dolomites. Dissertationes Botanicae 281, Cramer, Berlin: $197 \mathrm{pp}$.

Giacomini, V. \& S. Pignatti. 1955. Flora e vegetazione dell'Alta Valle del Braulio, con speciale riferimento ai pascoli di altitudine. Mem. Soc. Ital. Sci. Nat., 11: 47-238.

Giacomini, V., A. Pirola \& E. Wikus. 1962. I pascoli dell'alta Valle di S. Giacomo (Spluga). Flora et vegetatio italica mem. 4. Gianasso, Sondrio: $115 \mathrm{pp}$.

Gordon, N.D., T.A. McMahon, B.L. Finlayson, C.J. Gippel \& R.J. Nathan. 2004. Stream Hydrology: An Introduction for Ecologists. 2nd ed. Wiley \& Sons, New York: 444 pp.

Grabherr, G. \& L. Mucina 1993. Natürliche waldfreie Vegetation. In: Grabherr, G. \& L. Mucina (Eds), Die Pflanzengesellschaften Österreichs. Teil II. Fischer, Jena: 523 pp.

Guinochet, M. 1938. Etudes sur la végétation de l'étage alpin dans le bassin supérieur de la Tineé. Comm. S.I.G.M.A., Montpellier, 59: 1-458.

Hébrard, J.P. 1973. Contribution à l'étude de quelques bryoassociations de l'étage subalpin dans le sud-est de la France. Vegetatio, 27 (4-6): 347-381.

Herter, W. 1990. Die Pflanzengesellschaften des Hintersteiner Tales. Dissertationes Botanicae 147, Cramer, Berlin: 124 pp.

Hill, M.O., N. Bell, M.A. Bruggeman-Nannenga, M. Brugués, M.J. Cano, J. Enroth, K.I. Flatberg, J.P. Frahm, M.T. Gallego, R. Garilleti, J. Guerra, L. Hedenäs, D.T. Holyoak, J. Hyvönen, M.S. Ignatov, F. Lara, V. Mazimpaka, J. Muñoz \& L. Söderström. 2006. An annotated checklist of the mosses of Europe and Macaronesia. $J$. Bryol., 28 (3): 198-267.

Hinterlang, D. 1992. Vegetationsökologie der Weichwasserquellgesellschaften zentraleuropäischer Mittelgebirge. Crunoecia, 1: 1-117.

Kopecký, K. \& S. Hejný. 1978. Die Anwendung einer "deduktiven Methode syntaxonomische Klassification" bei der Bearbeitung der strassenbegeleitenden Pflanzengesellschaften Nordostböhmens. Vegetatio, 36 (1): 43-51.

Legendre, P. \& L. Legendre. 1998. Numerical ecology. Second English edition. Elsevier Science, B.V., Amsterdam: $853 \mathrm{pp}$.

Maarel, E. van der. 1979. Transformation of cover-abundance values in phytosociology and its effects on community similarity. Vegetatio, 39: 97-144.

Maas, F.M. 1959. Bronnen, bronbeken en bronbossen van Nederland, in het bijzonder die van de Veluwezoon. Meded. Landbouwhogesch. Wageningen, 59: 1-166.

Marstaller, R. 1987. Die Moosgesellschaften der Klasse Platyhypnidio-Fontinalietea antipyreticae Philippi 1956. Phytocoenologia, 15(1): 85-138.

Miserere, L. \& G. Buffa. 2001. Contributo alla conoscenza della vegetazione delle sorgenti calcaree in Valle d'Aosta. Braun-Blanquetia, 31: 27-32.

Mucina, L., G. Grabherr \& T. Ellmauer. 1993. Anthropogene Vegetation. In: Grabherr, G. \& L. Mucina (Eds), Die Pflanzengesellschaften Österreichs. Teil I. Fischer, Jena: $578 \mathrm{pp}$.

Philippi, G. \& E. Oberdorfer. 1992. Klasse: Montio-Cardaminetea. In: Oberdorfer, E. (Ed.), Süddeutsche Pflanzengesellschaften. Teil I, 3 Aufl. Fischer, Jena: 199-213.

Podani, L. 2001. Syn-Tax 2000. Computer Program for Data Analysis in Ecology and Systematics. Scientia Publishing, Budapest.

Privitera, M. \& M. Puglisi. 2004. La vegetazione briofitica della Sicilia. Braun-Blanquetia, 34: 129-141.

Reynolds, J.D. 1998. Ireland's freshwaters. Dublin, Marine Institute: $130 \mathrm{pp}$.

Ros R.M., V. Mazimpaka, U. Abou-Salama, M. Aleffi, T.L. Blockeel, M. Brugués, M.J. Cano, R.M. Cros, M.G. Dia, G.M. Dirkse, W. El Saadawi, A. Erdağ, A. Ganeva, J.M. Gonzáles-Macebo, I. Herrnstadt, K. Khalil, H. Kürschner, E. Lanfranco, A. Losada-Lima, M.S. Refai, S. RodríguezNuñez, M. Sabovljevic, C. Sergio, H. Shabbara, M. SimSim \& L. Söderström. 2007. Hepatics and Anthocerotes of the Mediterranean, an annotated checklist. Crypt. Bryol., 28: 351-437.

Schmidt, C. 1993. Die Wassermoosvegetation im Bergland Westfalens. Abh. Westf. Mus. Nat.kd. Münster, 4: 1-51.

Spitale, D. 2007. Assessing the ecomorphology of mountain springs: suggestions from a survey in the South-eastern Alps. In: Cantonati, M., E. Bertuzzi \& D. Spitale (Eds), The spring habitat: Biota and sampling methods. Monografie del Museo Tridentino di Scienze Naturali, 4: 31-44.

Spitale, D., A. Petraglia \& M. Tomaselli. 2009. Structural equation model detects unexpected differences between bryophyte and vascular plant richness along multiple environmental gradients. J. Biogeog., 36: 745-755.

Theurillat, J.P. 1992. Etude et cartographie du paysage végétal (symphytocoenologie) dans la région d'Aletsch (Valais, Suisse). Beitr. Geobot. Landesaufn. Schweiz 68, Flück-Wirth, Teufel: 384 pp.

Tomaselli, M. 2007. Vascular flora and vegetation in springs of the Alps: approaches to their investigation. In: Cantonati, M., E. Bertuzzi \& D. Spitale (Eds), The spring habitat: Biota and sampling methods. Monografie del Museo Tridentino di Scienze Naturali, 4: 137-146.

Thienemann, A. 1922. Hydrobiologische Untersuchungen an Quellen. (I-IV). Arch. Hydrobiol., 14: 151-190.

Warnke, A. \& A. Bogenrieder. 1985. Die Rieselfluren und Flachmoore der Feldberggruppe. Untersuchungen zur 
Temperatur, Sauerstoffsättigung und Ionenführung der Quellen am Feldberg. Ber. Naturforsch. Ges. Freib., 75: 91-124.

Weber, H. E., J. Moravec \& J. P. Theurillat. 2000. International Code of Phytosociological Nomenclature. 3rd ed. J. Veg. Sci., 11: 739-768.

Westhoff, V. \& E. Van der Maarel. 1973. The Braun-Blanquet approach. In: Whittaker R.H. (Ed.), Classification of plant communities. Junk, the Hague: 626-828.
Wikus, E. 1960. Die Vegetation der Lienzer Dolomiten (Osttirol). Arch. Bot. Biogeogr. Ital., 34-37: 1-189.

Wilmanns, O. 1989. Ökologische Pflanzensoziologie. 4. Aufl. Quelle \& Meyer, Heidelberg: 382 pp.

Zechmeister, H. 1993. Montio-Cardaminetea. In: Grabherr G. \& Mucina L. (Eds), Die Pflanzengesellschaften Österreichs. Teil II. Fischer, Jena: 213-240.

Zechmeister, H. \& L. Mucina. 1994. Vegetation of European springs: High-rank syntaxa of the Montio-Cardaminetea. J. Veg. Sci., 5 (3): 385-402. 